\title{
ÉTALE FUNDAMENTAL GROUPS OF KAWAMATA LOG TERMINAL SPACES, FLAT SHEAVES, AND QUOTIENTS OF ABELIAN VARIETIES
}

\author{
DANIEL GREB, STEFAN KEBEKUS, AND THOMAS PETERNELL
}

\section{CONTENTS}

1. Introduction 1

2. Main result 6

\section{Part I. Preparations 8}

3. Notation, conventions, and facts used in the proof 8

4. Chern classes on singular varieties 11

5. Bertini-type theorems for sheaves and their moduli 14

Part II. Quasi-étale covers of klt spaces 16

6. Proof of Theorems 2.1 and 1.1 16

7. Direct applications 18

8. Flat sheaves on klt base spaces $\quad 21$

9. Varieties with vanishing Chern classes 23

10. Varieties admitting polarised endomorphisms 25

11. Examples, counterexamples, and sharpness of results 26

\section{Appendices $\quad 29$}

Appendix A. Zariski's Main Theorem in the equivariant setting 29

Appendix B. Galois closure $\quad 31$

References $\quad 31$

\section{INTRODUCTION}

Working with a singular complex algebraic variety $X$, one is often interested in comparing the set of finite étale covers of $X$ with that of its smooth locus $X_{\text {reg. }}$. More precisely, one may ask the following.

What are the obstructions to extending finite étale covers of $X_{\text {reg }}$ to $X$ ? How do the étale fundamental groups of $X$ and of its smooth locus differ?

Date: 12th January 2016.

2010 Mathematics Subject Classification. 14J17, 14B05, 14E30, $14 \mathrm{~B} 25$.

Key words and phrases. Minimal Model Program, Algebraic Fundamental Group, KLT Singularities, Flat Vector Bundles, Torus Quotients, Polarised Endomorphisms.

All three authors were supported in part by the DFG-Forschergruppe 790 "Classification of Algebraic Surfaces and Compact Complex Manifolds". Daniel Greb gratefully acknowledges the support of the Baden-Württemberg-Stiftung through the "Eliteprogramm für Postdoktorandinnen und Postdoktoranden" and by the Institute of Mathematics at Albert-Ludwigs-Universität Freiburg. Stefan Kebekus acknowledges the support through a joint fellowship of the Freiburg Institute of Advanced Studies (FRIAS) and the University of Strasbourg Institute for Advanced Study (USIAS). 
We answer these questions for projective varieties $X$ with Kawamata log terminal (klt) singularities, a class of varieties that is important in the Minimal Model Program. The main result, Theorem 2.1, asserts that in any infinite tower of finite Galois morphisms over a klt variety, where all morphisms are étale in codimension one, almost every morphism is étale. In a certain sense, this result can be seen as saying that the difference between the sets of étale covers of $X$ and of $X_{\text {reg }}$ is small in case $X$ is klt. As an immediate application, we construct in Theorem $1.5 \mathrm{a}$ finite covering $\widetilde{X} \rightarrow X$, étale in codimension one, such that the étale fundamental groups of $\widetilde{X}$ and of its smooth locus $\widetilde{X}_{\text {reg }}$ agree. Further direct applications concern global bounds for the index of $\mathbb{Q}$-Cartier divisors on klt spaces, the existence of a quasi-étale "simultaneous index-one cover" where all Q-Cartier divisors are Cartier, and a finiteness result for étale fundamental groups of varieties of weak log Fano-type.

As a first major application, we obtain an extension theorem for flat vector bundles on klt varieties: after passing to a finite cover, étale in codimension one, any flat holomorphic bundle, defined on the smooth part of a klt variety extends across the singularities, to a flat bundle that is defined on the whole space. As a consequence, we show that every variety with terminal singularities and with vanishing first and second Chern class is a finite quotient of an Abelian variety, with a quotient map that is étale in codimension one. In the smooth case, this is a classical result, which follows from the existence of Kähler-Einstein metrics on Ricci-flat compact Kähler manifolds, due to Yau: every Ricci-flat compact Kähler manifold $X$ with $c_{2}(X)=0$ is an étale quotient of a compact complex torus. As a further application, we verify a conjecture of Nakayama and Zhang concerning varieties admitting polarised endomorphisms. Building on their results, we obtain a decomposition theorem describing the structure of these varieties.

1.1. Simplified version of the main result. Our main result, Theorem 2.1 , is quite general and its formulation is therefore somewhat involved. For many applications the following special case suffices.

Theorem 1.1. Let $X$ be a normal, complex, quasi-projective variety. Assume that there exists a $\mathbb{Q}$-Weil divisor $\Delta$ such that $(X, \Delta)$ is Kawamata log terminal (klt). Assume we are given a sequence of finite, surjective morphisms of normal varieties that are étale in codimension one,

$$
X=Y_{0} \longleftarrow \gamma_{1} Y_{1} \longleftarrow \gamma_{2} Y_{2} \longleftarrow \gamma_{3} Y_{3} \longleftarrow \gamma_{4} \cdots .
$$

If the composed morphisms $\gamma_{1} \circ \cdots \circ \gamma_{i}: Y_{i} \rightarrow X$ are Galois for every $i \in \mathbb{N}^{+}$, then all but finitely many of the morphisms $\gamma_{i}$ are étale.

Remark 1.2 (Galois morphisms). In Theorem 1.1 and throughout this paper, Galois morphisms are assumed to be finite and surjective, but need not be étale; see Definition 3.6. The statement of Theorem 1.1 does not continue to hold when one drops the Galois assumption. Section 11.1 discusses an example that illustrates the problems in the non-Galois setting.

Remark 1.3. In accordance with the notation of Kollár-Kovács [KK10] one might call varieties $X$ "potentially klt" if they admit a divisor $\Delta$ that makes the pair $(X, \Delta)$ klt. For sake of brevity, we will later call quasi-finite, surjective morphisms which are étale in codimension one "quasi-étale"; see Definition 3.3, a terminology which was first introduced by F. Catanese in [Cat07].

Remark 1.4 (Purity of branch locus). By purity of the branch locus, the assumption that all morphisms $\gamma_{i}$ of Theorem 1.1 are étale in codimension one can also be formulated in one of the following, equivalent ways. 
(1.4.1) All morphisms $\gamma_{1} \circ \cdots \circ \gamma_{i}$ are étale over the smooth locus of $Y_{0}$.

(1.4.2) Given any $i \in \mathbb{N}^{+}$, then $\gamma_{i}$ is étale over the smooth locus of $Y_{i-1}$.

1.2. Direct Applications. Theorems 1.1 and 2.1 have a large number of immediate consequences. As a first direct application, we show that every klt space admits a quasi-étale cover whose étale fundamental group equals that of its smooth locus.

Theorem 1.5 (Extension of étale covers from the smooth locus of klt spaces). Let $X$ be a normal, complex, quasi-projective variety. Assume that there exists a $\mathbb{Q}$-Weil divisor $\Delta$ such that $(X, \Delta)$ is klt. Then, there exists a normal variety $\widetilde{X}$ and a finite, surjective Galois morphism $\gamma: \widetilde{X} \rightarrow X$, étale in codimension one, such that the following equivalent conditions hold.

(1.5.1) Any finite, étale cover of $\widetilde{X}_{\text {reg }}$ extends to a finite, étale cover of $\widetilde{X}$.

(1.5.2) The natural map $\widehat{\iota}_{*}: \widehat{\pi}_{1}\left(\widetilde{X}_{\text {reg }}\right) \rightarrow \widehat{\pi}_{1}(\widetilde{X})$ of étale fundamental groups induced by the inclusion of the smooth locus, $\iota: \widetilde{X}_{\text {reg }} \rightarrow \widetilde{X}$, is an isomorphism.

In fact, somewhat more general statements are true; cf. Section 7.1.1. To avoid any potential for confusion, we briefly recall the characterisation of the étale fundamental group of a complex variety.

Fact 1.6 (Étale fundamental group, [Mil80, $\S 5$ and references there]). If $Y$ is any complex algebraic variety, then the étale fundamental group $\widehat{\pi}_{1}(Y)$ is isomorphic to the profinite completion of the topological fundamental group of the associated complex space $Y^{a n}$.

Remark 1.7 (Reformulation of Theorem 1.5 in terms of étale fundamental groups). Although it might seem natural, Theorem 1.5 does not imply that the kernel of the natural map $\iota_{*}: \widehat{\pi}_{1}\left(X_{\text {reg }}\right) \rightarrow \widehat{\pi}_{1}(X)$ is finite. A counterexample is discussed in Section 11.2 on page 27. We do not know whether Theorem 1.5 can be expressed solely in terms of the étale fundamental groups $\widehat{\pi}_{1}\left(X_{\text {reg }}\right)$ and $\widehat{\pi}_{1}(X)$.

Remark 1.8 (Canonical choice of minimal $\widetilde{X}$ ). It is natural to ask whether there exists a canonical choice of $\widetilde{X}$, uniquely determined by a suitable minimality property. This is not the case. We will show in Section 11.3 that in general no "minimal cover" exists.

The following local variant of Theorem 1.5 considers coverings of neighbourhoods of a given point, rather than coverings of the full space.

Theorem 1.9 (Local version of Theorem 1.5). Let $X$ be a normal, complex, quasiprojective variety. Assume that there exists a $\mathbb{Q}$-Weil divisor $\Delta$ such that $(X, \Delta)$ is klt. Let $p \in X$ be any closed point. Then, there exists a Zariski-open neighbourhood $X^{\circ}$ of $p \in X$, a normal variety $\widetilde{X}^{\circ}$ and a finite, surjective Galois morphism $\gamma: \widetilde{X}^{\circ} \rightarrow X^{\circ}$, étale in codimension one, such that the following holds: given any Zariski-open neighbourhood $U=U(p) \subseteq X^{\circ}$ with preimage $\widetilde{U}:=\gamma^{-1}(U)$, then $\widehat{\pi}_{1}\left(\widetilde{U}_{\mathrm{reg}}\right) \cong \widehat{\pi}_{1}(\widetilde{U})$. Equivalently, any finite, étale cover of $\widetilde{U}_{\text {reg }}$ extends to a finite, étale cover of $\widetilde{U}$.

Among its many properties, the covering constructed in Theorem 1.9 can be seen as a simultaneous index-one cover for all divisors on $X^{\circ}$ that are $Q$-Cartier in a neighbourhood of $p$. In fact, the following much stronger result holds true.

Theorem 1.10 (Simultaneous index-one cover). In the setting of Theorem 1.9, the following holds for any Zariski-open neighbourhood $U=U(p) \subseteq X^{\circ}$ with preimage $\widetilde{U}=\gamma^{-1}(U)$.

(1.10.1) If $\widetilde{D}$ is any $Q$-Cartier divisor on $\widetilde{U}$, then $\widetilde{D}$ is Cartier.

(1.10.2) If $D$ is any $\mathbb{Q}$-Cartier divisor on $U$, then $(\# \mathrm{Gal}(\gamma)) \cdot D$ is Cartier. 
Remark 1.11 (Global bound for the index of Q-Cartier divisors on klt spaces). Under the assumptions of Theorem 1.9, it follows from (1.10.2) and from quasicompactness of $X$ that there exists a number $N \in \mathbb{N}^{+}$such that $N \cdot D$ is Cartier, whenever $D$ is a $Q$-Cartier divisor on $X$.

Remark 1.12. It seems to be known to experts that a covering space satisfying a weak analogue of (1.10.1) exists for spaces with rational singularities. In contrast to the usual index-one covers discussed in higher-dimensional birational geometry, the Galois group of the morphism $\gamma$ need not be cyclic.

As a last direct application of Theorem 1.5, we obtain a new proof of a recent result by Chenyang $\mathrm{Xu},[\mathrm{Xu14}, \mathrm{Thm} .2]$. Note that this is not independent of $\mathrm{Xu}$ 's work, as the proof of Theorem 1.5 uses [Xu14, Thm. 1]; however, we do not use [HMX14].

Theorem 1.13. Let $X$ be a normal, complex, projective variety. Assume that there exists $a \mathbb{Q}$-Weil divisor $\Delta$ such that $(X, \Delta)$ is $k l t$, and $-\left(K_{X}+\Delta\right)$ is big and nef. Then, the étale fundamental group $\widehat{\pi}_{1}\left(X_{\mathrm{reg}}\right)$ is finite.

In Section 11.2 we show by way of example that Theorem 1.13 cannot be generalised to rationally connected varieties.

1.3. Extension for flat sheaves on klt base spaces. Consider a normal variety $X$ and a flat, locally free, analytic sheaf $\mathscr{F}^{\circ}$, defined on the complex manifold $X_{\text {reg }}^{\text {an }}$ associated with the smooth locus $X_{\text {reg }}$ of $X$. In this setting, a fundamental theorem of Deligne, [Del70, II.5, Cor. 5.8 and Thm. 5.9], asserts that $\mathscr{F}^{\circ}$ is algebraic, and thus extends to a coherent, algebraic sheaf $\mathscr{F}$ on $X$. If $X$ is klt, we will show that Deligne's extended sheaf $\mathscr{F}$ is again locally free and flat, at least after passing to a quasi-étale cover.

Theorem 1.14 (Extension of flat, locally free sheaves). Let $X$ be a normal, complex, quasi-projective variety. Assume that there exists a $\mathbb{Q}$-Weil divisor $\Delta$ such that $(X, \Delta)$ is klt. Then, there exists a normal variety $\widetilde{X}$ and a finite, surjective Galois morphism $\gamma$ : $\widetilde{X} \rightarrow X$, étale in codimension one, such that the following holds. If $\mathscr{G}^{\circ}$ is any flat, locally free, analytic sheaf on the complex space $\widetilde{X}_{\mathrm{reg}}^{\text {an }}$, there exists a flat, locally free, algebraic sheaf $\mathscr{G}$ on $\widetilde{X}$ such that $\mathscr{G}^{\circ}$ is isomorphic to the analytification of $\left.\mathscr{G}\right|_{\widetilde{X}_{\mathrm{reg}}}$.

Theorem 1.14 follows as a consequence of Theorem 1.5. Except for the algebraicity assertion, we do not use Deligne's result in our proof. In order to avoid confusion, we briefly recall the definition of flat sheaves.

Definition 1.15 (Flat locally free sheaf). If $Y$ is any complex algebraic variety, and $\mathscr{G}$ is any locally free, analytic sheaf on the underlying complex space $Y^{\text {an }}$, we call $\mathscr{G}$ flat if it is defined by a representation of the topological fundamental group $\pi_{1}\left(Y^{a n}\right)$. A locally free, algebraic sheaf on $Y$ is called flat if and only if the associated analytic sheaf is flat.

Using the partial confirmation of the Lipman-Zariski conjecture shown in [GKKP11], we obtain the following criterion for a klt space to have quotient singularities. We also obtain a first criterion to guarantee that a given projective variety is a quotient of an Abelian variety.

Corollary 1.16 (Criterion for quotient singularities and torus quotients). In the setting of Theorem 1.14, if $\mathscr{T}_{X_{\text {reg }}}$ is flat, then $\widetilde{X}$ is smooth and $X$ has only quotient singularities. If $X$ is additionally assumed to be projective, then there exists an Abelian variety $A$ and a finite Galois morphism $A \rightarrow X$ that is étale in codimension one. 
1.4. Characterisation of torus quotients: varieties with vanishing Chern classes. Consider a Ricci-flat, compact Kähler manifold $X$ whose second Chern class vanishes. As a classical consequence of Yau's theorem [Yau78] on the existence of a Kähler-Einstein metric, $X$ is then covered by a complex torus; cf. [LB70, Thm. 12.4.3] and [Kob87, Ch. IV, Cor. 4.15]. Building on our main result, we generalise this to the singular case, when $X$ has terminal or a special type of klt singularities.

Theorem 1.17 (Characterisation of torus quotients). Let $X$ be a normal, complex, projective variety of dimension $n$ with at worst klt singularities. Assume that $X$ is smooth in codimension two and that the canonical divisor is numerically trivial, $K_{X} \equiv 0$. Further, assume that there exist ample divisors $H_{1}, \ldots, H_{n-2}$ on $X$ and a desingularisation $\pi$ : $\widetilde{X} \rightarrow X$ such that $c_{2}\left(\mathscr{T}_{\tilde{X}}\right) \cdot \pi^{*}\left(H_{1}\right) \cdots \pi^{*}\left(H_{n-2}\right)=0$.

Then, there exists an Abelian variety $A$ and a finite, surjective, Galois morphism $A \rightarrow$ $X$ that is étale in codimension two.

In fact, the converse is also true; see Theorem 9.3 for a precise statement. The three-dimensional case has been settled by Shepherd-Barron and Wilson in [SBW94], and our strategy of proof for Theorem 1.17 partly follows their line of reasoning. Apart from our main result, the proof of Theorem 1.17 relies on the semistability of the tangent sheaf of varieties with vanishing first Chern class, on Simpson's flatness results for semistable sheaves, [Sim92, Cor. 3.10], and on the partial solution of the Lipman-Zariski conjecture mentioned above.

Remark 1.18 (Vanishing of Chern classes). The condition on the intersection numbers posed in Theorem 1.17 is a way of saying " $c_{2}(X)=0$ " that avoids the technical complications with the definition of Chern classes on singular spaces. Section 4 discusses this in detail.

Remark 1.19 (Terminal varieties, Generalisations). Projective varieties with terminal singularities are smooth in codimension two, [KM98, Cor. 5.18]. Theorem 1.17 therefore applies to this class of varieties. From the point of view of the minimal model program, this seems a very natural setting for our problem.

Using an orbifold version of the second Chern class, Shepherd-Barron and Wilson [SBW94] are able to treat threefolds whose singular set has codimension two. It is conceivable that with sufficient technical work, using our line of argumentation a similar result could also be obtained in the higher-dimensional setting. We have chosen not to pursue these generalisations here.

As one important step in the proof of Theorem 1.17, we generalise classical flatness results [UY86, Kob87, Sim92, BS94] for semistable vector bundles with vanishing first and second Chern classes to our singular setup, and obtain the following result:

Theorem 1.20 (Flatness of semistable sheaves with vanishing first and second Chern classes). Let $X$ be an n-dimensional, normal, complex, projective variety, smooth in codimension two. Assume that there exists a $\mathrm{Q}$-Weil divisor $\Delta$ such that $(X, \Delta)$ is klt. Let $H$ be an ample Cartier divisor on $X$, and $\mathscr{E}$ be a reflexive, $H$-slope-semistable sheaf. Assume that the following intersection numbers vanish

$$
c_{1}(\mathscr{E}) \cdot H^{n-1}=0, \quad c_{1}(\mathscr{E})^{2} \cdot H^{n-2}=0, \quad \text { and } \quad c_{2}(\mathscr{E}) \cdot H^{n-2}=0 .
$$

Then, there exists a normal variety $\widetilde{X}$ and a finite, surjective Galois morphism $\gamma: \widetilde{X} \rightarrow X$, étale in codimension one, such that $\left(\gamma^{*} \mathscr{E}\right)^{* *}$ is locally free and flat, that is, $\left(\gamma^{*} \mathscr{E}\right)^{* *}$ is given by a linear representation of $\pi_{1}(\widetilde{X})$. 
1.5. Characterisation of torus quotients: varieties admitting polarised endomorphisms. In [NZ10], Nakayama and Zhang study the structure of varieties admitting polarised endomorphisms - the notion of "polarised endomorphism" is recalled in Remark 1.22 below. They conjecture in [NZ10, Conj. 1.2] that any variety of this kind is either uniruled or covered by an Abelian variety, with a covering map that is étale in codimension one. The conjecture has been shown in special cases, for instance in dimensions less than four. As an immediate application of Theorem 1.1, we show that it holds in full generality.

Theorem 1.21 (Varieties with polarised endomorphisms; cf. [NZ10, Conj. 1.2]). Let $X$ be a normal, complex, projective variety admitting a non-isomorphic polarised endomorphism. Assume that $X$ is not uniruled. Then, there exists an Abelian variety $A$ and $a$ finite, surjective morphism $A \rightarrow X$ that is étale in codimension one.

Remark 1.22 (Polarised endomorphism). Let $X$ be a normal, complex, projective variety. An endomorphism $f: X \rightarrow X$ is called polarised if there exists an ample Cartier divisor $H$ and a positive number $q \in \mathbb{N}^{+}$such that $f^{*}(H) \sim q \cdot H$.

Theorem 1.21 has strong implications for the structure of varieties with endomorphisms. These are discussed in Section 10.2 on page 26.

1.6. Outline of the paper. Section 2 formulates and discusses the main results. Before proving these in Part II, we have gathered in Part I a number of results and facts which will later be used in the proofs. While many of the facts discussed in Sections 3 and 5 are known to experts (though hard to find in the literature), the material of Section 4 is new to the best of our knowledge and might be of independent interest.

The main result and its applications are proven in Part II. The statements are somewhat delicate and might invite misinterpretation unless care is taken. We have therefore chosen to conclude with a number of examples in Section 11, showing that the assumptions are strictly necessary and that several "obvious" generalisations or reformulations are wrong. The concluding appendices prove uniqueness and equivariance in "Zariski's Main Theorem in the form of Grothendieck" and invariance of branch loci under Galois closure.

1.7. Acknowledgements. The authors would like to thank Hélène Esnault, Carlo Gasbarri, Patrick Graf, Annette Huber-Klawitter, James McKernan, Wolfgang Soergel, and Matthias Wendt for numerous discussions. Chenyang $\mathrm{Xu}$ kindly answered our questions by e-mail. Angelo Vistoli and Jason Starr responded to Stefan Kebekus' questions on the MathOverflow web site. We are grateful to De-Qi Zhang for his interest in our work and for pointing us towards [Amb05]. Moreover, we thank Jochen Heinloth for discussions concerning boundedness of flat sheaves and for providing references from [Sim94]. The authors are particularly grateful to Fritz Hörmann, who found a mistake in the first preprint version of this paper. Finally, the authors would like to thank an anonymous referee for pointing out several simplifications in the proof of the main result.

\section{MAIN RESULt}

The introductory section presented a simplified version of our main result. The following more general Theorem 2.1 differs from the simplified version in two important aspects, which we briefly discuss to prepare the reader for the somewhat technical formulation. Figure 2.1 on the facing page illustrates the setup schematically. 


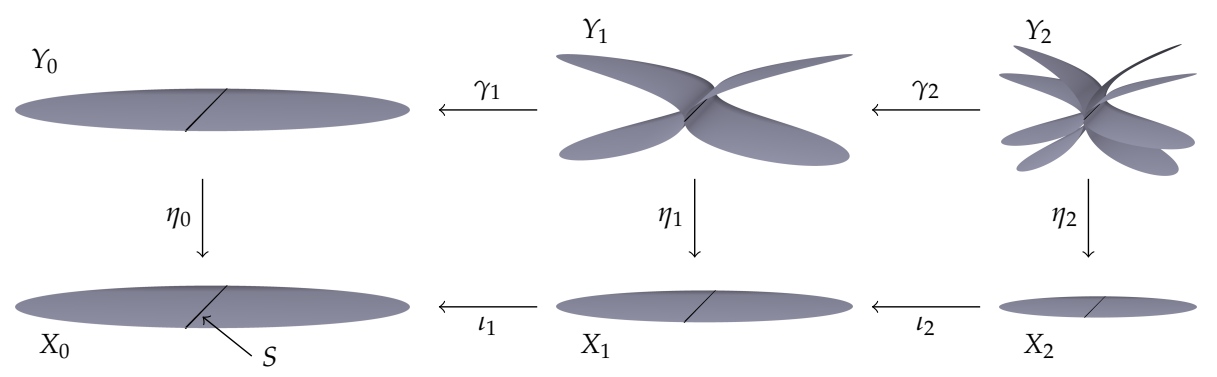

The figure shows the setup for the main result, Theorem 2.1, schematically. The morphisms $\eta_{i}$ are Galois covers over a sequence $X \supseteq X_{0} \supseteq X_{1} \supseteq \cdots$ is increasingly small open subsets of $X$. The morphisms $\gamma_{i}$ between these covering spaces are étale away from the preimages of $S$. In Theorem 2.1, the set $S$ is of codimension two or more. This aspect is difficult to illustrate and therefore not properly shown in the figure.

FIGURE 2.1. Setup for the main result

Aspect 1: Finite vs. quasi-finite morphisms. For simplicity, we have assumed in Theorem 1.1 that all morphisms $\gamma_{i}$ are finite. However, there are settings where this assumption is too restrictive and where one would like to consider quasi-finite rather than finite morphisms. The proofs of Theorems 1.9 and 1.10 provide examples for this more general setup.

To support this kind of application, Theorem 2.1 allows the morphisms $\gamma_{i}$ to be quasi-finite, as long as each $Y_{i}$ is Galois over a suitable open subset $X_{i} \subseteq X$. For this reason, Theorem 2.1 introduces a descending chain of dense open subsets $X \supseteq X_{0} \supseteq X_{1} \supseteq \cdots$ and replaces Sequence (1.1.1) by the more complicated Diagram (2.1.1).

Aspect 2: Specification of the branch locus. The morphisms $\gamma_{i}$ of Theorem 1.1 are required to be étale in codimension one, and therefore branch only over the singular locus of the target varieties $Y_{i-i}$. However, there are settings where it is advantageous to specify the potential branch locus in a more restrictive manner, requiring that the $\gamma_{i}$ branch only over a given set $S$. This gives more precise information on the branch locus.

Theorem 2.1 (Main result). Let $X$ be a normal, complex, quasi-projective variety of dimension $\operatorname{dim} X \geq 2$. Assume that there exists a $\mathbb{Q}$-Weil divisor $\Delta$ such that $(X, \Delta)$ is klt. Suppose further that we are given a descending chain of dense open subsets $X \supseteq$ $X_{0} \supseteq X_{1} \supseteq \cdots$, a closed reduced subscheme $S \subset X$ of codimension $\operatorname{codim}_{X} S \geq 2$, and a commutative diagram of morphisms between normal varieties,

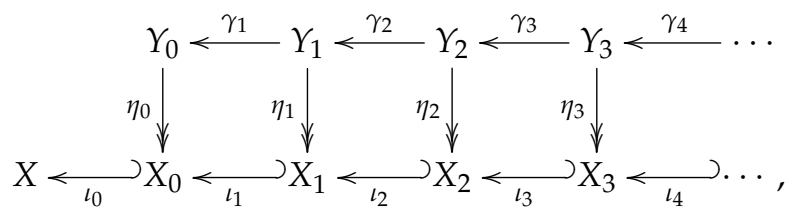

where the following holds for all indices $i \in \mathbb{N}$.

(2.1.2) The morphisms $l_{i}$ are the inclusion maps.

(2.1.3) The morphisms $\gamma_{i}$ are quasi-finite, dominant and étale away from the reduced preimage set $S_{i}:=\eta_{i}^{-1}(S)_{\text {red. }}$.

(2.1.4) The morphisms $\eta_{i}$ are finite, surjective, Galois, and étale away from $S_{i}$.

Then, all but finitely many of the morphisms $\gamma_{i}$ are étale. 
Remark 2.2 (Interdependence of conditions in Theorem 2.1). Conditions (2.1.3) and (2.1.4) are not independent. The assumption that the morphisms $\gamma_{i}$ are étale away from $S_{i}$ follows trivially from Condition (2.1.4) and [Mil80, Cor. 3.6]. We have chosen to include the redundancy for convenience of reference and notation.

\section{Part I. Preparations}

\section{NOTATION, CONVENTIONS, AND FACTS USED IN THE PROOF}

3.1. Global conventions. Throughout this paper, all schemes, varieties and morphisms will be defined over the complex number field. We follow the notation and conventions of Hartshorne's book [Har77]. In particular, varieties are always assumed to be irreducible. For complex spaces and holomorphic maps, we follow [GR84] whenever possible. For all notations around Mori theory, such as klt spaces and klt pairs, we refer the reader to [KM98]. The empty set has dimension minus infinity, $\operatorname{dim} \varnothing=-\infty$.

3.2. Notation. In the course of the proofs, we frequently need to switch between the Zariski and the Euclidean topology. We will consistently use the following notation.

Notation 3.1 (Complex space associated with a variety). Given a variety or projective scheme $X$, denote by $X^{\text {an }}$ the associated complex space, equipped with the Euclidean topology. If $f: X \rightarrow Y$ is any morphism of varieties or schemes, denote the induced map of complex spaces by $f^{a n}: X^{a n} \rightarrow Y^{a n}$. If $\mathscr{F}$ is any coherent sheaf of $\mathscr{O}_{X}$-modules, denote the associated coherent analytic sheaf of $\mathscr{O}_{X}{ }^{a n}$-modules by $\mathscr{F}^{\text {an }}$.

Definition 3.2 (Covers and covering maps, compare with Definition 3.4). Let $Y$ be a normal, connected variety or complex space. A cover of $Y$ is a surjective, finite morphism (resp. holomorphic map) $f: X \rightarrow Y$, where $X$ is again normal and connected. The map $f$ is called a covering map. Two covers $f: X \rightarrow Y$ and $f^{\prime}: X^{\prime} \rightarrow Y$ are called isomorphic if there exists an isomorphism (resp. biholomorphic map) $\psi: X \rightarrow X^{\prime}$ such that $f^{\prime} \circ \psi=f$. In other words, the following diagram should commute:

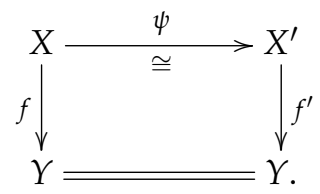

Note that in Definition 3.2 we do not assume $f$ to be étale. Morphisms that are étale in codimension one will also be called "quasi-étale".

Definition 3.3 (Quasi-étale morphisms). A morphism $f: X \rightarrow Y$ between normal varieties is called quasi-étale if $f$ is quasi-finite and étale in codimension one. In other words, $f$ is quasi-étale if $\operatorname{dim} X=\operatorname{dim} Y$ and if there exists a closed, subset $Z \subseteq X$ of codimension $\operatorname{codim}_{X} Z \geq 2$ such that $\left.f\right|_{X \backslash Z}: X \backslash Z \rightarrow Y$ is étale.

Definition 3.4 (Finite topological covering space; cf. [Hat02, Sect. 1.3]). Given any topological space $Y$, a finite topological covering space is a topological space $X$ together with a surjective, continuous map $\gamma: X \rightarrow Y$ such that there exists an open cover $\left\{U_{\alpha}\right\}_{\alpha \in A}$ of $Y$ with the property that for each $\alpha \in A$, the preimage $\gamma^{-1}\left(U_{\alpha}\right)$ is a finite disjoint union of open sets in $X$, each of which is mapped homeomorphically onto $U_{\alpha}$ by $\gamma$.

Remark 3.5 (Comparison with Definition 3.2). In contrast to Definition 3.2, we do not assume that covering spaces are connected. A morphism of varieties that is a cover in the sense of Definition 3.2 is a finite topological covering space if and only if it is étale. 
3.3. Galois morphisms. Galois morphisms appear prominently in the literature, but their precise definition is not consistent. We will use the following definition.

Definition 3.6 (Galois morphism). A covering map $\gamma: X \rightarrow Y$ of varieties is called Galois if there exists a finite group $G \subset \operatorname{Aut}(X)$ such that $\gamma$ is isomorphic to the quotient map.

It is a standard, widely-used fact that any finite, surjective morphism between normal varieties can be enlarged to become Galois.

Theorem 3.7 (Existence of Galois closure). Let $\gamma: X \rightarrow Y$ be a covering map of quasiprojective varieties. Then, there exists a normal, quasi-projective variety $\widetilde{X}$ and a finite, surjective morphism $\widetilde{\gamma}: \widetilde{X} \rightarrow X$ such that the following holds.

(3.7.1) There exist finite groups $H \leqslant G$ such that the morphisms $\Gamma:=\gamma \circ \tilde{\gamma}$ and $\widetilde{\gamma}$ are Galois with group $G$ and $H$, respectively.

(3.7.2) If $\operatorname{Branch}(\gamma)$ and $\operatorname{Branch}(\Gamma)$ denote the branch loci, with their natural structure as reduced subschemes of $Y$, then $\operatorname{Branch}(\gamma)=\operatorname{Branch}(\Gamma)$.

The morphism $\Gamma: \widetilde{X} \rightarrow Y$ is often called the Galois closure of $\gamma$. A proof of Theorem 3.7 is given in Appendix B.

3.4. Zariski's Main Theorem. Grothendieck observed in [Gro66] that Zariski's Main Theorem can be reformulated by saying that any quasi-finite morphism decomposes as a composition of an open immersion and a finite map. If all varieties in question are normal, this decomposition is unique and well-behaved with respect to the action of the relative automorphism group.

Theorem 3.8 (Zariski's Main Theorem in the equivariant setting). Let $a: V^{\circ} \rightarrow$ $W$ be any quasi-finite morphism between quasi-projective varieties. Assume that $V^{\circ}$ is normal and not the empty set. Then there exists a normal, quasi-projective variety $V$ and $a$ factorisation of a into an open immersion $\alpha: V^{\circ} \rightarrow V$ and a finite morphism $\beta: V \rightarrow W$. The factorisation is unique up to unique isomorphism and satisfies the following additional conditions with respect to group actions.

(3.8.1) If $G$ is any group that acts on $V^{\circ}$ and $W$ by algebraic morphisms, and if $a$ is equivariant with respect to these actions, then $G$ also acts on $V$ and the morphisms $\alpha$ and $\beta$ are equivariant with respect to these actions.

(3.8.2) If $W^{\circ}:=\operatorname{Image}(a)$ is open in $W$ and $a: V^{\circ} \rightarrow W^{\circ}$ is Galois with group $G$, then $\beta: V \rightarrow W$ is Galois with group $G$.

Remark 3.9 (Algebraic group actions). If the group $G$ in (3.8.1) is algebraic and acts algebraically on $V^{\circ}$, then the induced action on $V$ will clearly also be algebraic.

In the affine setting, parts of Theorem 3.8 are found in [Dré04]. A full proof of Theorem 3.8 is given in Appendix A.

3.5. Covers of stratified spaces. In this section, we investigate the interplay of branch loci with Whitney stratifications of the base space. We follow the terminology of Goresky-MacPherson's book [GM88]. The following proposition summarises material from [GM88, Thm. in I.1.7 and I.1.4].

Proposition 3.10 (Whitney stratification of algebraic varieties and subvarieties). Let $X$ be an algebraic variety and $A \subsetneq X$ a Zariski-closed subset.

(3.10.1) There exists a finite Whitney stratification $\left(X_{i}\right)_{i \in \Lambda}$ of $X$ into disjoint, locally closed, smooth subvarieties $X_{i}$ of $X$ such that $A$ is a union of strata.

(3.10.2) Given any Whitney stratification $\left(X_{i}\right)_{i \in \Lambda}$ as in (3.10.1) and any $i \in \Lambda$, then $X_{i}$ has a neighbourhood $U_{i}$ in $X^{\text {an }}$ that admits a continuous, locally trivial projection $f_{i}: U_{i} \rightarrow X_{i}^{a n}$. Given any $p \in X_{i}$, the fibre $f_{i}^{-1}(p)$ is homeomorphic to the cone over the link of $X_{i}$ and therefore 1-connected. 
Remark 3.11. Given any Whitney stratification $\left(X_{i}\right)_{i \in \Lambda}$ as in (3.10.1) and any Zariski-open $V \subseteq X$, observe that $\left(X_{i} \cap V\right)_{i \in \Lambda}$ is again a Whitney stratification of $V$. In the setting of (3.10.2), observe that the identity $X_{i}^{a n} \rightarrow X_{i}^{a n} \subseteq U_{i}$ yields a section of $f_{i}$. The restriction $\left.f_{i}\right|_{U_{i} \backslash X_{i}^{a n}}$ is likewise locally trivial.

Corollary 3.12. In the setting of Proposition 3.10, assume that $\operatorname{codim}_{X} A \geq 2$ and let $\gamma: Y \rightarrow X$ be a covering map whose branch locus is contained in $A$. If $i \in \Lambda$ is any index such that the Zariski-closure $\overline{X_{i}}$ equals a component of $A$, then either the branch locus contains $X_{i}$ or it is disjoint from $X_{i}$.

Proof. Given $a \in X_{i} \backslash \operatorname{Branch}(\gamma)$, we need to show that $X_{i} \cap \operatorname{Branch}(\gamma)=\varnothing$. Removing strata, we are free to assume that $X_{i}=A$. Write

$$
U_{i}^{\circ}:=U_{i} \backslash X_{i}, \quad Y^{\circ}:=\left(\gamma^{a n}\right)^{-1}\left(U_{i}^{\circ}\right), \quad F:=f_{i}^{-1}(a), \quad F^{\circ}:=F \cap U_{i}^{\circ} .
$$

Observe that $U_{i}^{\circ}$ and $F$ do not intersect the the branch locus. Consider the long exact homotopy sequences,

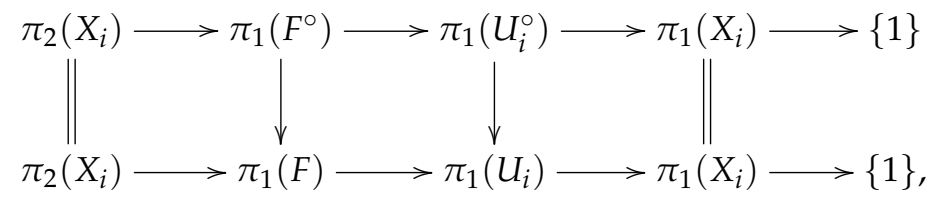

where the vertical arrows are induced by the inclusions.

Choose $q \in F^{\circ}$. The finite topological covering space $Y^{\circ} \rightarrow U_{i}^{\circ}$ corresponds to an action of $\pi_{1}\left(U_{i}^{\circ}\right)$ on the finite set $Q:=\gamma^{-1}(q)$, [Hat02, p. 68ff]. Since $\gamma$ is unbranched over the 1-connected set $F$, observe that the induced action of $\pi_{1}\left(F^{\circ}\right)$ is trivial. The action of $\pi_{1}\left(U_{i}^{\circ}\right)$ is therefore induced by an action of $\pi_{1}\left(X_{i}\right)$, hence by an action of $\pi_{1}\left(U_{i}\right)$. It follows that $\left.\gamma^{a n}\right|_{Y^{\circ}}$ can be extended to a finite topological covering space $\gamma^{\prime}: Y^{\prime} \rightarrow X$, which carries the structure of a normal complex space, locally biholomorphic to $X$. But the two covering spaces $Y^{a n}$ and $Y^{\prime}$ agree outside of $A$, which is of codimension two, and are therefore isomorphic by [DG94, Thm. 3.4]. In particular, $\gamma$ is locally biholomorphic.

3.6. Classification of holomorphic covering maps. It is a standard result of topology that covering spaces of a given topological space are classified by conjugacy classes of the fundamental group. Using fundamental results of Grauert, Remmert, and Stein this statement can be generalised to branched holomorphic coverings of a given normal complex space.

Proposition 3.13 (Classification of covering maps with prescribed branch locus). Let $X$ be a connected, normal complex space and $A \subsetneq X$ a proper analytic subset. Then the following natural map is bijective:

$$
\left.\begin{array}{c}
\left\{\begin{array}{c}
\text { Isomorphism classes of } \\
\text { holomorphic covering maps } \\
f: Y \rightarrow X \text {, where } f \text { is local- } \\
\text { ly biholom. away from } f^{-1}(A)
\end{array}\right\} \\
f
\end{array} \quad \rightarrow \quad \begin{array}{c}
\text { Finite-index subgroups of } \\
\widehat{\pi}_{1}(X \backslash A) \text { up to conjugation }
\end{array}\right\}
$$

Remark 3.14 (Equivalence of categories). The map of Proposition 3.13 induces an equivalence of categories, but we will not need this stronger statement.

Proof of Proposition 3.13. The classification of finite connected topological covering spaces of $X \backslash A$, [Hat02, Thm. 1.38], and the fact that any such cover can be given a uniquely determined complex structure that makes the covering map 
holomorphic and locally biholomorphic, [DG94, § 1.3], together establish a correspondence between isomorphism classes of locally biholomorphic covering maps $f^{\circ}: Y^{\circ} \rightarrow X \backslash A$ and conjugacy classes of finite-index subgroups of $\widehat{\pi}_{1}(X \backslash A)$, by sending $f^{\circ}$ to $\left(f^{\circ}\right)_{*} \widehat{\pi}_{1}\left(Y^{\circ}\right)$.

In order to prove our claim, it therefore suffices to show that any locally biholomorphic holomorphic covering map $f^{\circ}: Y^{\circ} \rightarrow X \backslash A$ can be uniquely extended to a holomorphic covering map $f: Y \rightarrow X$, where $Y$ is a connected normal complex space. This however is a special case of [DG94, Thm. 3.4]; for this, observe that the assumption made in [DG94, Thm. 3.4] on the analyticity of $A \cup B$ is automatically fulfilled in our setup, since the critical locus of the covering that we aim to extend is empty.

Remark 3.15. Theorem [DG94, Thm. 3.4] is a modern version of results obtained by Grauert-Remmert and Stein in their fundamental papers [GR58, Ste56] dating back to the 1950's.

Corollary 3.16 (Characterisation of biholomorphic maps). Consider a sequence of holomorphic covering maps, $W_{2} \stackrel{\gamma}{\rightarrow} W_{1} \stackrel{\eta}{\rightarrow} V$. Let $A \subsetneq V$ be any a proper analytic subset such that $\eta$ and $\eta \circ \gamma$ are locally biholomorphic away from $A$. Then $\gamma$ is biholomorphic if and only if the following two subgroups of $\widehat{\pi}_{1}(V \backslash A)$ agree up to conjugation,

$$
\eta_{*} \widehat{\pi}_{1}\left(W_{1} \backslash \eta^{-1}(A)\right) \text { and }(\eta \circ \gamma)_{*} \widehat{\pi}_{1}\left(W_{2} \backslash(\eta \circ \gamma)^{-1}(A)\right) \text {. }
$$

Proof. If $\gamma$ is biholomorphic, then $W_{1} \backslash \eta^{-1}(A)$ and $W_{2} \backslash(\eta \circ \gamma)^{-1}(A)$ are isomorphic over $V \backslash A$. This implies that the two subgroups $\eta_{*} \widehat{\pi}_{1}\left(W_{1} \backslash \eta^{-1}(A)\right)$ and $(\eta \circ \gamma)_{*} \widehat{\pi}_{1}\left(W_{2} \backslash(\eta \circ \gamma)^{-1}(A)\right)$ agree up to conjugation.

Now assume that $\eta_{*} \widehat{\pi}_{1}\left(W_{1} \backslash \eta^{-1}(A)\right)$ and $(\eta \circ \gamma)_{*} \widehat{\pi}_{1}\left(W_{2} \backslash(\eta \circ \gamma)^{-1}(A)\right)$ are conjugate. Then, Proposition 3.13 implies that the covering maps $\eta$ and $\eta \circ \gamma$ are isomorphic. In other words, there exists a biholomorphic map $\mu: W_{1} \rightarrow W_{2}$ such that $(\eta \circ \gamma) \circ \mu=\eta$. For any point $p \in V \backslash A$, we therefore have $\left|(\eta \circ \gamma)^{-1}(p)\right|=$ $\left|\eta^{-1}(p)\right|$. Hence, the degree of $\gamma$ is equal to one. As $W_{1}$ is normal, the analytic version of Zariski's Main Theorem implies that $\gamma$ is biholomorphic, as claimed.

\section{CHERN CLASSES ON SINGULAR VARIETIES}

4.1. Intersection numbers on singular varieties. To prove the characterisation of torus quotients given in Theorem 1.17, we need to discuss intersection numbers of line bundles with Chern classes of reflexive sheaves on varieties with canonical singularities. For our purposes, it is actually not necessary to define Chern classes themselves. The literature discusses several competing notions of Chern classes on singular spaces, all of which are technically challenging; cf. [Mac74, Alu06]. For the reader's convenience, we have chosen to include a short, self-contained presentation, restricting ourselves to the minimal material required for the proof.

Definition 4.1 (Resolution of a space and a coherent sheaf). Let $X$ be a normal variety and $\mathscr{E}$ a coherent sheaf of $\mathscr{O}_{X}$-modules. A resolution of $(X, \mathscr{E})$ is a proper, birational and surjective morphism $\pi: \widetilde{X} \rightarrow X$ such that the space $\widetilde{X}$ is smooth, and such that the sheaf $\pi^{*}(\mathscr{E}) /$ tor is locally free. If $\pi$ is isomorphic over the open set where $X$ is smooth and $\mathscr{E} /$ tor is locally free, we call $\pi$ a strong resolution of $(X, \mathscr{E})$.

Remark 4.2. In the setup of Definition 4.1, the existence of a resolution of singularities combined with a classical result of Rossi, [Ros68, Thm. 3.5], shows that resolutions and strong resolutions of $(X, \mathscr{E})$ exist.

Definition 4.3 (Intersection of Chern class with Cartier divisors). Let X be a normal, $n$-dimensional, quasi-projective variety and $\mathscr{E}$ be a coherent sheaf of $\mathscr{O}_{X}$-modules. Assume 
we are given a number $i \in \mathbb{N}^{+}$such that $X$ is smooth in codimension $i$ and such that $\mathscr{E}$ is locally free in codimension $i$. Given any resolution morphism $\pi: \widetilde{X} \rightarrow X$ of $(X, \mathscr{E})$ and any set of Cartier divisors $L_{1}, \ldots L_{n-i}$ on $X$, we use the following shorthand notation

$$
c_{i}(\mathscr{E}) \cdot L_{1} \cdots L_{n-i}:=c_{i}(\mathscr{F}) \cdot\left(\pi^{*} L_{1}\right) \cdots\left(\pi^{*} L_{n-i}\right) \in \mathbb{Z} .
$$

where $\mathscr{F}:=\pi^{*} \mathscr{E} /$ tor, and where $c_{i}(\mathscr{F})$ denotes the classical Chern class of the locally free sheaf $\mathscr{F}$ on the smooth variety $\widetilde{X}$. More generally, if $P\left(y_{1}, \ldots, y_{n}\right)$ is a homogeneous polynomial of degree i for weighted variables $y_{j}$ with $\operatorname{deg} y_{j}=j$, we will use the shorthand notation

$$
P\left(c_{1}(\mathscr{E}), \ldots, c_{n}(\mathscr{E})\right) \cdot L_{1} \cdots L_{n-i}:=P\left(c_{1}(\mathscr{F}), \ldots, c_{n}(\mathscr{F})\right) \cdot\left(\pi^{*} L_{1}\right) \cdots\left(\pi^{*} L_{n-i}\right) .
$$

Remark 4.4 (Independence of resolution). In the setting of Definition 4.3, given another smooth variety $\widehat{X}$ and a diagram

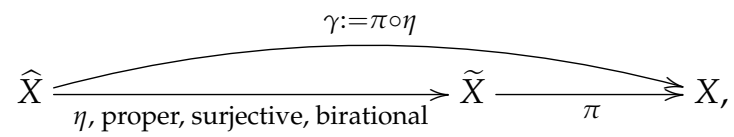

then $\left(\gamma^{*} \mathscr{E}\right) /$ tor $\cong \eta^{*}\left(\left(\pi^{*} \mathscr{E}\right) /\right.$ tor $)$. It follows immediately from the projection formula for Chern classes, [Ful98, Thm. 3.2 on p. 50], that

$$
c_{i}\left(\left(\pi^{*} \mathscr{E}\right) / \text { tor }\right) \cdot\left(\pi^{*} L_{1}\right) \cdots\left(\pi^{*} L_{n-i}\right)=c_{i}\left(\left(\gamma^{*} \mathscr{E}\right) / \text { tor }\right) \cdot\left(\gamma^{*} L_{1}\right) \cdots\left(\gamma^{*} L_{n-i}\right) .
$$

The same holds for the more complicated expressions involving the polynomial $P$. Since any two resolution maps are dominated by a common third, the numbers defined in Definition 4.3 are independent of the choice of the resolution map.

Remark 4.5 (Alternative computation for semiample divisors). In the setup of Definition 4.3, assume we are given numbers $m_{1}, \ldots, m_{n-i} \in \mathbb{N}^{+}$and basepointfree linear systems $B_{j} \subseteq\left|m_{j} L_{j}\right|$, for all $1 \leq j \leq n-i$. Choose general elements $\Delta_{j} \in B_{j}$ and consider the intersection

$$
S:=\Delta_{1} \cap \cdots \cap \Delta_{n-i} .
$$

Observe that $S$ is smooth, entirely contained in the smooth locus $X_{\text {reg }} \subseteq X$, that $\mathscr{E}$ is locally free along $S$, and that

$$
P\left(c_{1}(\mathscr{E}), \ldots, c_{n}(\mathscr{E})\right) \cdot L_{1} \cdots L_{n-i}=\frac{P\left(c_{1}\left(\left.\mathscr{E}\right|_{S}\right), \ldots, c_{n}\left(\left.\mathscr{E}\right|_{S}\right)\right)}{m_{1} \cdots m_{n-i}} \in \mathbb{Z},
$$

where the left hand side is given as in Definition 4.3 above.

The following proposition is a fairly direct consequence of Remark 4.5 and the projection formula.

Proposition 4.6 (Behaviour under covers, étale in codimension $i$ ). In the setup of Definition 4.3, if $\gamma: \widetilde{X} \rightarrow X$ is any cover, étale in codimension $i$, then $\widetilde{X}$ is smooth in codimension $i$, the sheaf $\gamma^{*} \mathscr{E}$ is locally free in codimension $i$, and

$$
\begin{aligned}
(\operatorname{deg} \gamma) \cdot P\left(c_{1}(\mathscr{E}), \ldots, c_{n}(\mathscr{E})\right) & \cdot L_{1} \cdots L_{n-i} \\
= & P\left(c_{1}\left(\gamma^{*} \mathscr{E}\right), \ldots, c_{n}\left(\gamma^{*} \mathscr{E}\right)\right) \cdot\left(\gamma^{*} L_{1}\right) \cdots\left(\gamma^{*} L_{n-i}\right) .
\end{aligned}
$$

Proof. The statements about smoothness of $\widetilde{X}$ and about local freeness of $\gamma^{*} \mathscr{E}$ follow from purity of the branch locus.

Since any Cartier divisor is linearly equivalent to the difference of two very ample divisors, we can assume without loss of generality that the divisors $L_{1}, \ldots, L_{n-i}$ are very ample and general in basepoint-free linear systems. The intersection $S:=L_{1} \cap \cdots \cap L_{n-i}$ is then smooth, entirely contained in $X_{\text {reg, }}$ and the morphism $\gamma$ is étale near $S$. Writing $\widetilde{S}:=\gamma^{-1}(S)=\gamma^{*} L_{1} \cap \cdots \cap \gamma^{*} L_{n-i}$, observe 
that the alternative computation of Remark 4.5 applies to both $S$ and $\widetilde{S}$, and yields the following.

$$
\begin{aligned}
P\left(c_{1}\left(\gamma^{*} \mathscr{E}\right), \ldots,\right. & \left.c_{n}\left(\gamma^{*} \mathscr{E}\right)\right) \cdot\left(\gamma^{*} L_{1}\right) \cdots\left(\gamma^{*} L_{n-i}\right) & & \\
& =P\left(c_{1}\left(\left.\gamma^{*} \mathscr{E}\right|_{\widetilde{S}}\right), \cdots, c_{n}\left(\left.\gamma^{*} \mathscr{E}\right|_{\widetilde{S}}\right)\right) & & \text { Remark } 4.5 \text { for } \widetilde{X} \\
& =\operatorname{deg} \gamma \cdot P\left(c_{1}\left(\left.\mathscr{E}\right|_{S}\right), \ldots, c_{n}\left(\left.\mathscr{E}\right|_{S}\right)\right) & & \text { Projection Formula } \\
& =\operatorname{deg} \gamma \cdot P\left(c_{1}(\mathscr{E}), \ldots, c_{1}(\mathscr{E})\right) \cdot L_{1} \cdots L_{n-i} & & \text { Remark } 4.5 \text { for } X .
\end{aligned}
$$

This finishes the proof of Proposition 4.6.

4.2. Numerically trivial Chern classes. Theorem 1.17 discusses varieties $X$ where the intersection numbers $c_{2}\left(\mathscr{T}_{X}\right) \cdot H_{1} \cdots H_{n-2}$ vanish for certain ample divisors $H_{1}, \ldots, H_{n-2}$. We will see in Proposition 4.8 that this sometimes implies that all intersection numbers with arbitrary divisors vanish. The following definition is relevant in the discussion.

Definition 4.7 (Numerically trivial Chern class). In the setting of Definition 4.3, we say that $c_{i}(\mathscr{E})$ is numerically trivial, if $c_{i}(\mathscr{E}) \cdot L_{1} \cdots L_{n-i}=0$ for all Cartier divisors $L_{1}, \ldots, L_{n-i}$ on $X$.

Proposition 4.8 (Criterion for numerical triviality of $c_{2}\left(\mathscr{T}_{X}\right)$ ). Let $X$ be a normal, n-dimensional, projective variety that is smooth in codimension two. Assume that the canonical divisor $K_{X}$ is Q-Cartier and nef. Then, $c_{2}\left(\mathscr{T}_{X}\right)$ is numerically trivial if and only if there exist ample Cartier divisors $H_{1}, \ldots, H_{n-2}$ on $X$ such that

$$
c_{2}\left(\mathscr{T}_{X}\right) \cdot H_{1} \cdots H_{n-2}=0
$$

Before proving Proposition 4.8 in Section 4.3 below, we note the following immediate corollary of Propositions 4.6 and 4.8 .

Corollary 4.9 (Behaviour of numerically trivial $c_{2}\left(\mathscr{T}_{X}\right)$ under coverings). Let $X$ be a normal, $n$-dimensional, projective variety that is smooth in codimension two. Assume that the canonical divisor $K_{X}$ is Q-Cartier and nef. If $\gamma: X^{\prime} \rightarrow X$ is any cover, étale in codimension two, then $X^{\prime}$ is smooth in codimension two, and $K_{X^{\prime}}$ is Q-Cartier and nef. Moreover, $c_{2}\left(\mathscr{T}_{X}\right)$ is numerically trivial if and only if $c_{2}\left(\mathscr{T}_{X^{\prime}}\right)$ is numerically trivial.

4.3. Proof of Proposition 4.8. The proof of Proposition 4.8 spans the current Section 4.3. For the convenience of the reader, we have subdivided the proof into several relatively independent steps.

Step 1: Setup of notation. Let $H_{1}, \ldots, H_{n-2}$ be ample Cartier divisors on $X$ such that (4.8.1) holds. Let further Cartier divisors $L_{1}, \ldots, L_{n-2}$ be given, and let $\pi$ : $\widetilde{X} \rightarrow X$ be a strong resolution of singularities. To prove Proposition 4.8 , we need to show that

$$
c_{2}\left(\mathscr{T}_{X}\right) \cdot L_{1} \cdots L_{n-2}=0 .
$$

Since each of the $L_{i}$ can be written as a difference of very ample divisors, it suffices to show the vanishing under the additional assumption that each of the $L_{i}$ is ample. Replacing the $H_{i}$ with sufficiently high powers, we can even assume without loss of generality that the following holds.

Assumption w.l.o.g. 4.10. For any index $i$, the divisors $L_{i}, H_{i}+L_{i}$ and $H_{i}-L_{i}$ are ample. 
Step 2: Miyaoka semipositivity. In order to prove (4.9.1) we shall make use of Miyaoka's Semipositivity Theorem. In our setup, it asserts that the intersection numbers of $c_{2}\left(\mathscr{T}_{X}\right)$ with nef Cartier divisors on $X$ are never negative.

Claim 4.11 (Miyaoka semipositivity). Assumptions as above. If $A_{1}, \ldots, A_{n-2}$ are ample Cartier divisors on $X$, then $c_{2}\left(\mathscr{T}_{X}\right) \cdot A_{1} \cdots A_{n-2} \geq 0$.

Proof. Let $S \subset X$ be a complete intersection surface, as introduced in Remark 4.5. Observe that $S$ avoids the singularities of $X$, and that the strong resolution map $\pi$ is isomorphic along $S$. Since $\pi^{*}\left(\mathscr{T}_{X}\right)$ and $\mathscr{T}_{\tilde{X}}$ differ only along the $\pi$-exceptional set, Remark 4.5 shows that

$$
c_{2}\left(\mathscr{T}_{X}\right) \cdot A_{1} \cdots A_{n-2}=c_{2}\left(\mathscr{T}_{\tilde{X}}\right) \cdot\left(\pi^{*} A_{1}\right) \cdots\left(\pi^{*} A_{n-2}\right)
$$

Using the assumption that the canonical divisor $K_{X}$ is Q-Cartier and nef, Miyaoka shows in [Miy87, Thm. 6.6] that the right hand side is always non-negative.

As a first corollary of Claim 4.11 we obtain the following technical result, which shows vanishing of a larger class of intersection numbers.

Consequence 4.12. Assumptions as above. For all numbers $1 \leq k \leq n-2$, we have the following vanishing of numbers,

$$
c_{2}\left(\mathscr{T}_{X}\right) \cdot\left(H_{1}+L_{1}\right) \cdots\left(H_{k}+L_{k}\right) \cdot H_{k+1} \cdots H_{n-2}=0 .
$$

Proof. We prove Consequence 4.12 by induction on $k$. For $k=0$, Equation (4.12.1) equals Equation (4.8.1), which holds by assumption. For the inductive step, assume that Equation (4.12.1) holds for a given number $k$. We need to show that it holds for $k+1$. To this end, consider the following two computations,

$$
\begin{aligned}
0 & \leq c_{2}\left(\mathscr{T}_{X}\right) \cdot\left(H_{1}+L_{1}\right) \cdots\left(H_{k}+L_{k}\right) \cdot\left(H_{k+1} \pm L_{k+1}\right) \cdot H_{k+2} \cdots H_{n-2} \\
& = \pm c_{2}\left(\mathscr{T}_{X}\right) \cdot\left(H_{1}+L_{1}\right) \cdots\left(H_{k}+L_{k}\right) \cdot L_{k+1} \cdot H_{k+2} \cdots H_{n-2} .
\end{aligned}
$$

The Inequalities (4.12.2) hold by Claim 4.11, because the two bundles $H_{k+1} \pm L_{k+1}$ are both ample by Assumption 4.10. The Equalities (4.12.3) hold by induction hypothesis. Together, the inequalities show that the two numbers computed must both be zero. This finishes the proof of Consequence 4.12.

Step 3: End of proof. To prove Equation (4.9.1) and thereby finish the proof of Proposition 4.8, apply Consequence 4.12 for $k=n-2$. We obtain the equation

$$
c_{2}\left(\mathscr{T}_{X}\right) \cdot\left(H_{1}+L_{1}\right) \cdots\left(H_{n-2}+L_{n-2}\right)=0 .
$$

Multiplying out, we write this number as a sum of the form

$$
0=\sum_{j} c_{2}\left(\mathscr{T}_{X}\right) \cdot A_{1, j} \cdots A_{n-2, j} \quad \text { where } A_{i, j} \in\left\{H_{i}, L_{i}\right\} \text { for all indices } i .
$$

Recalling that the divisors $H_{i}$ and $L_{i}$ are ample, Claim 4.11 thus asserts that none of the summands is negative. Summing up to zero, we obtain that all summands must actually be zero. To finish the proof of Proposition 4.8, observe that $c_{2}\left(\mathscr{T}_{X}\right)$. $L_{1} \cdots L_{n-2}$ is one of the summands involved.

\section{BERTINI-TYPE THEOREMS FOR SHEAVES AND THEIR MODULI}

The proofs of our main results use the fact that two reflexive sheaves belonging to a bounded family are isomorphic if and only if their restrictions to a general hyperplane of sufficiently high degree agree. The present section is devoted to the proof of this auxiliary result.

Proposition 5.1 (Testing isom. classes of reflexive sheaves on hyperplanes). Let $X$ be a normal, projective variety of dimension $\operatorname{dim} X \geq 2$ and $\mathscr{E}, \mathscr{F}$ two coherent, reflexive sheaves of $\mathscr{O}_{X}$-modules. Let $H \subset X$ be an irreducible, reduced, ample Cartier divisor on $X$ such that the following holds. 
(5.1.1) The variety $H$ is normal.

(5.1.2) Setting $\mathscr{H}:=\mathscr{H}$ om $(\mathscr{E}, \mathscr{F})$, the restricted sheaves $\left.\mathscr{E}\right|_{H},\left.\mathscr{F}\right|_{H}$ and $\left.\mathscr{H}\right|_{H}$ are reflexive.

(5.1.3) Let $X^{\prime} \subsetneq X$ be the minimal closed set such that $\mathscr{E}$ and $\mathscr{F}$ are locally free outside of $X^{\prime}$. Setting $H^{\prime}:=X^{\prime} \cap H$, we have $\operatorname{codim}_{H} H^{\prime} \geq 2$.

(5.1.4) The cohomology group $H^{1}\left(X, \mathscr{I}_{H} \otimes \mathscr{H}\right)$ vanishes.

Then, $\mathscr{E}$ is isomorphic to $\mathscr{F}$ if and only if the sheaves $\left.\mathscr{E}\right|_{H}$ and $\left.\mathscr{F}\right|_{H}$ are isomorphic.

A proof is given below. As a consequence, we obtain the following result.

Proposition 5.2 (Bertini-type theorem for isom. classes of reflexive sheaves). Let $X$ be a normal, projective variety of dimension $\operatorname{dim} X \geq 2$ and $\mathscr{E}, \mathscr{F}$ two coherent, reflexive sheaves of $\mathscr{O}_{X}$-modules. If $\mathscr{L} \in \operatorname{Pic}(X)$ is ample, then there exists a number $M \in \mathbb{N}$ and for any $m \geq M$ a dense open subset $V_{m} \subseteq\left|\mathscr{L}^{\otimes m}\right|$ such that all hyperplanes $H \in V_{m}$ are reduced and normal, and such that $\mathscr{E} \cong \mathscr{F}$ if and only if $\left.\left.\mathscr{E}\right|_{H} \cong \mathscr{F}\right|_{H}$.

Proof. Consider the sheaf $\mathscr{H}:=\mathscr{H} o m(\mathscr{E}, \mathscr{F})$. Since $\mathscr{F}$ is assumed to be reflexive, so is $\mathscr{H}$. Since $X$ is normal, $\mathscr{H}$ hence has depth $\geq 2$ at every point of $X$, [Har80, Prop. 1.3]. It hence follows from [Gro68, Exp. XII, Prop. 1.5] that

$$
H^{1}\left(X,\left(\mathscr{L}^{*}\right)^{\otimes m} \otimes \mathscr{H}\right)=0 \text { for all } m \gg 0 .
$$

Choose one $M \in \mathbb{N}$ such that Equation (5.2.1) holds for all $m \geq M$, and such that the linear systems $\left|\mathscr{L}^{\otimes m}\right|$ are basepoint-free.

To prepare for the construction of $V_{m}$, recall from [Har80, Cor. 1.4] that $\mathscr{E}, \mathscr{F}$ and $\mathscr{H}$ are locally free outside of a closed subset $X^{\prime} \subsetneq X$ with $\operatorname{codim}_{X} X^{\prime} \geq 2$. Now, given any number $m \geq M$, let $V_{m} \subseteq\left|\mathscr{L}^{\otimes m}\right|$ be the maximal open set such that the following holds for all hyperplanes $H \in V_{m}$.

(5.2.2) The hyperplane $H$ is irreducible, reduced and normal.

(5.2.3) The intersection $H^{\prime}:=H \cap X^{\prime}$ is small, that is, $\operatorname{codim}_{H} H^{\prime} \geq 2$.

(5.2.4) The restricted sheaves $\left.\mathscr{E}\right|_{H},\left.\mathscr{F}\right|_{H}$ and $\left.\mathscr{H}\right|_{H}$ are reflexive.

Seidenberg's theorem, [BS95, Thm. 1.7.1], Bertini's theorem, and [Gro66, Thm. 12.2.1] guarantee that none of the open sets $V_{m}$ is empty. Together with (5.2.1), Proposition 5.1 therefore applies to all $H \in V_{m}$.

Let $X$ be a normal projective variety of dimension $\operatorname{dim} X \geq 2$, let $\mathscr{E}$ be a coherent, reflexive sheaf of $\mathscr{O}_{X}$-modules, and $F$ be a bounded family of locally free sheaves. If $H$ is any sufficiently ample Cartier divisor, then

$$
H^{1}\left(X, \mathscr{J}_{H} \otimes \mathscr{H} \text { om }(\mathscr{E}, \mathscr{F})\right)=0 \quad \text { for all } \mathscr{F} \in F \text {. }
$$

Note that $\left.\mathscr{F}\right|_{H}$ is locally free for all $\mathscr{F} \in F$ and for all hyperplanes $H \in\left|\mathscr{L}^{\otimes m}\right|$. With the same arguments as above, an iterative application of Proposition 5.1 therefore also yields the following.

Corollary 5.3 (Iterated Bertini-type theorem for bounded families). Let $X$ be a normal, projective variety of dimension $\operatorname{dim} X \geq 2$. Let $\mathscr{E}$ be a coherent, reflexive sheaf of $\mathscr{O}_{X^{-}}$ modules, and let $F$ be a bounded family of locally free sheaves. Given an ample line bundle $\mathscr{L} \in \operatorname{Pic}(X)$, a sufficiently increasing sequence $0 \ll m_{1} \ll m_{2} \ll \cdots \ll m_{k}$ and general elements $H_{i} \in\left|\mathscr{L}^{\otimes m_{i}}\right|$ with associated complete intersection variety $S:=H_{1} \cap \cdots \cap H_{k}$, then the following holds for all sheaves $\mathscr{F} \in F$. The sheaf $\mathscr{F}$ is isomorphic to $\mathscr{E}$ if and only if $\left.\mathscr{F}\right|_{S}$ is isomorphic to $\mathscr{E} \mid s$.

Proof of Proposition 5.1. The implication " $\left.\left.\mathscr{E} \cong \mathscr{F} \Rightarrow \mathscr{E}\right|_{H} \cong \mathscr{F}\right|_{H}$ " is clear. For the opposite direction assume for the remainder of the proof that we are given isomorphism $\lambda_{H}:\left.\left.\mathscr{E}\right|_{H} \rightarrow \mathscr{F}\right|_{H}$. Using the vanishing (5.1.4), we aim to extend $\lambda_{H}$ to a morphism $\lambda: \mathscr{E} \rightarrow \mathscr{F}$, which will turn out to be isomorphic. 
Step 1: Restriction of $\mathscr{H}$. We compare the restriction $\left.\mathscr{H}\right|_{H}$ to the homomorphism sheaf associated with the restrictions, $\left.\mathscr{H}_{H}:=\left.\mathscr{H} O \mathscr{H}_{(\mathscr{E}}\right|_{H},\left.\mathscr{F}\right|_{H}\right)$. Note that the latter has a non-trivial section given by $\lambda_{H}$. Item (5.1.2) implies that $\left.\mathscr{H}\right|_{H}$ and $\mathscr{H}_{H}$ are reflexive sheaves of $\mathscr{O}_{H}$-modules. It is also clear that outside of the small set $H^{\prime} \subset H$ the sheaves $\left.\mathscr{H}\right|_{H}$ and $\mathscr{H}_{H}$ are both locally free and agree. But since two reflexive sheaves are isomorphic if and only if they are isomorphic on the complement of a small set, this immediately yields an isomorphism $\left.\mathscr{H}\right|_{H} \cong \mathscr{H}_{H}$.

Step 2: Extension of the morphism $\lambda_{H}$. We will now show that there exists a morphism $\lambda: \mathscr{E} \rightarrow \mathscr{F}$ such that $\lambda_{H}=\left.\lambda\right|_{H}$. To this end, consider the ideal sheaf sequence of the hypersurface $H$, that is, $0 \rightarrow \mathscr{J}_{H} \rightarrow \mathscr{O}_{X} \rightarrow \mathscr{O}_{H} \rightarrow 0$. Since $\mathscr{H}$ is torsion free, this sequence stays exact when tensoring with $\mathscr{H}$, [Wei94, Sect. 3.1]. The associated long exact sequence cohomology sequence then reads

$$
\cdots \rightarrow H^{0}(X, \mathscr{H}) \stackrel{r}{\rightarrow} H^{0}\left(X,\left.\mathscr{H}\right|_{H}\right) \rightarrow \underbrace{H^{1}\left(X, \mathscr{J}_{H} \otimes \mathscr{H}\right)}_{=0 \text { by }(5.1 .4)} \rightarrow \cdots,
$$

where $r$ is the natural restriction map. We have seen in Step 1 that the middle term in (5.3.1) is isomorphic to $H^{0}\left(X, \mathscr{H}_{H}\right)$, which contains $\lambda_{H}$. Surjectivity of $r$ therefore implies that $\lambda_{H}$ can be extended. Choose one extension $\lambda$ and fix this choice throughout. To finish the proof, we need to show that $\lambda$ is an isomorphism.

Step 3: Injectivity of $\lambda$. Both kernel and image of the morphism $\lambda$ are subsheaves of the torsion free sheaves $\mathscr{E}$ and $\mathscr{F}$, respectively, and therefore themselves torsion free. Let $X^{\circ} \subseteq X$ be the maximal open subset, where $\operatorname{ker}(\lambda), \mathscr{E}$ and $\operatorname{img}(\lambda)$ are locally free. Recalling from [Har80, Cor. 1.4] that $\operatorname{codim}_{X} X \backslash X^{\circ} \geq 2$, the intersection $H^{\circ}:=H \cap X^{\circ}$ is clearly non-empty, and the restricted sequence

$$
\left.\left.\left.0 \rightarrow \operatorname{ker}(\lambda)\right|_{H^{\circ}} \rightarrow \mathscr{E}\right|_{H^{\circ}} \stackrel{\left.\lambda\right|_{H^{\circ}}}{\longrightarrow} \operatorname{img}(\lambda)\right|_{H^{\circ}} \rightarrow 0
$$

remains exact. Since $\left.\lambda\right|_{H^{\circ}}=\left.\lambda_{H}\right|_{H^{\circ}}$ is isomorphic, the sheaf $\left.\operatorname{ker}(\lambda)\right|_{H^{\circ}}$ vanishes, showing that the morphism $\lambda$ is injective in an open neighbourhood of $H^{\circ}$. Since $\mathscr{E}$ is reflexive, hence torsion free, it follows that $\lambda$ is injective.

Step 4: Surjectivity of $\lambda$, end of proof. Restriction to $H$ is a functor that is exact on the right. It follows that $\left.\operatorname{coker}(\lambda)\right|_{H}=\operatorname{coker}\left(\left.\lambda\right|_{H}\right)=0$. In particular, we see that the support of $\operatorname{coker}(\lambda)$ does not intersect the hyperplane $H$. Since $H$ is ample, it follows that the support of $\operatorname{coker}(\lambda)$ is finite, and that the injective map $\lambda$ is isomorphic away from this finite set. Using the standard fact that two reflexive sheaves are isomorphic if and only if they are isomorphic away from a small set, it follows that $\lambda$ is an isomorphism. This finishes the proof of Proposition 5.2.

\section{Part II. Quasi-étale covers of klt spaces}

\section{Proof of THEOREMS 2.1 AND 1.1}

6.1. Proof of Theorem 2.1. Maintaining notation and assumptions of Theorem 2.1, write $\delta_{j, i}:=\gamma_{i+1} \circ \cdots \circ \gamma_{j}: Y_{j} \rightarrow Y_{i}$. Recall from [Har77, II Cor. 4.8.e] that $\delta_{j, i}$ is finite over $\eta_{i}^{-1}\left(X_{j}\right)$ and write

$$
T_{i}:=\overline{\bigcup_{j>i} \operatorname{Branch} \delta_{j, i}} \subseteq S_{i} \subsetneq Y_{i} .
$$

We may assume that $T_{0} \neq \varnothing$. The proof proceeds by induction over $\operatorname{dim} T_{0}$. 
6.1.1. Start of Induction. Assume that $\operatorname{dim} T_{0}=0$. Choose a point $t \in T_{0}$. We may assume that $t \in \eta_{0}^{-1}\left(X_{i}\right)$ for all $i \in \mathbb{N}$, for otherwise there is nothing to show. Next, choose a descending sequence of analytically open neighbourhoods of $t \in Y_{0}^{a n}$, say $\left(V_{i}\right)_{i \in \mathbb{N}}$, such that the following holds.

(6.1.1) The set $V_{0}$ intersects $T_{0}$ precisely in $t$. The set $V_{0} \backslash\{t\}$ is homeomorphic to the open cone over the link $\operatorname{Link}\left(Y_{0}, t\right)$.

(6.1.2) For positive indices $i$, there are inclusions $V_{i} \subseteq \eta_{0}^{-1}\left(X_{i}\right)^{a n}$. The connected components of $\left(\delta_{i, 0}^{a n}\right)^{-1}\left(V_{i}\right)$ each contain exactly one point over $T_{0}$.

(6.1.3) The inclusion maps $V_{i} \backslash\{t\} \rightarrow V_{0} \backslash\{t\}$ are homotopy equivalences.

We refer the reader to [GR84, Sect. 2.3.2], [Dim92, Thm. 5.1] for the results used here. Next, choose a sequence of connected components, $W_{i} \subseteq\left(\delta_{i, 0}^{a n}\right)^{-1}\left(V_{i}\right)$ such that $\gamma_{i}^{a n}\left(W_{i}\right) \subseteq W_{i-1}$. Let $t_{i} \in W_{i}$ be the unique point lying over $t$. We obtain a descending sequence of subgroups,

$$
G_{i}:=\left(\delta_{i, 0}^{a n}\right)_{*} \widehat{\pi}_{1}\left(W_{i} \backslash\left\{t_{i}\right\}\right) \subseteq \widehat{\pi}_{1}\left(V_{i} \backslash\{t\}\right)=\widehat{\pi}_{1}\left(V_{0} \backslash\{t\}\right) .
$$

Remark 6.2. It follows from (2.1.4) that the morphisms $\delta_{i, 0}$ are Galois. If $i$ is any index and $W_{i}^{\prime} \neq W_{i}$ is any other connected component of $\left(\delta_{i, 0}^{a n}\right)^{-1}\left(V_{i}\right)$ with associated group $G_{i}^{\prime}$, then $G_{i}$ and $G_{i}^{\prime}$ are both normal, and in fact equal. The groups $G_{i}$ do therefore not depend on the specific choice of the $W_{i}$.

The descending sequence $\left(G_{i}\right)_{i \in \mathbb{N}^{+}}$stabilises because the algebraic local fundamental group $\hat{\pi}_{1}^{\text {loc }}(X, s):=\widehat{\pi}_{1}\left(\operatorname{Link}\left(Y_{0}, t\right)\right)$ of the klt base space $Y_{0}$ is finite, [Xu14, Thm. 1]. This allows us to choose $M \in \mathbb{N}$ such that $G_{i}=G_{M}$ for all $i>M$. It follows from Corollary 3.16 that the morphisms $\gamma_{i}$ are étale over $t$, for all $i>M$. This finishes the proof in case where $T_{0}$ is finite.

6.1.2. Inductive step. Assume that $\operatorname{dim} T_{0}>0$ and that Theorem 2.1 has already been shown for all diagrams with smaller-dimensional $T_{0}$ 's. Choose Whitney stratifications of the varieties $Y_{i}$ as in Proposition 3.10, such that the subsets $T_{i} \subsetneq Y_{i}$ are unions of strata. If $A \subsetneq X$ is a very general hyperplane, the following will hold.

(6.2.1) The hyperplane $A$ is irreducible, normal, not contained in supp $\Delta$, and $\left(A,\left.\Delta\right|_{A}\right)$ is klt.

(6.2.2) The set $S_{A}:=S \cap A$ has codimension at least two in $A$.

(6.2.3) For every $i \in \mathbb{N}$, the hyperplane $B_{i}:=\eta_{i}^{-1}(A)$ intersects every positivedimensional stratum of $Y_{i}$ non-trivially with the appropriate codimension. In particular, $B_{i} \not \subset T_{i}$ and $\operatorname{dim} B_{0} \cap T_{0}<\operatorname{dim} T_{0}$.

Write $A_{k}:=A \cap X_{k}$, note that is non-empty for all $k \geq 0$, and observe that the restriction of Diagram (2.1.1),

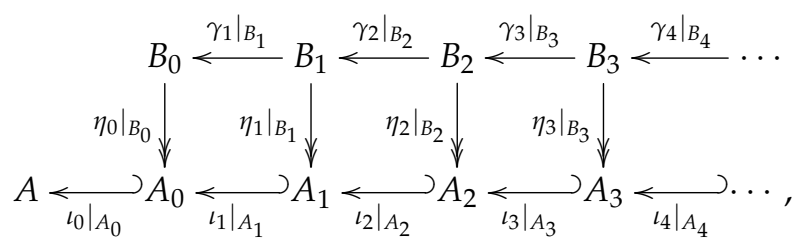

reproduces all assumptions made in Theorem 2.1. By induction hypothesis, there exists a number $M \in \mathbb{N}^{+}$such that the morphisms $\left.\gamma_{i}\right|_{B_{i}}$ are étale for all $i \geq M$. For simplicity of notation, we may assume without loss of generality that $M=1$. It thus follows immediately from the characterisation of étale morphisms in terms of fibre size, [Gro71, I. Thm. 10.11], that for any $i \geq 1$ the morphism $\gamma_{i}$ is étale in a neighborhood of $B_{i}$. Corollary 3.12 then implies that the branch locus of any of 
the composed morphisms $\delta_{i, 0}$ is contained in the set

$T_{0}^{\prime}:=T_{0} \backslash\left\{\right.$ union of strata that are dense in some component of $\left.T_{0}\right\}$, whose dimension is strictly less than $\operatorname{dim} T_{0}$.

6.2. Proof of Theorem 1.1. Under the assumptions of Theorem 1.1, set $S:=X_{\text {sing, }}$, $X_{i}:=X, \iota_{i}:=\operatorname{Id}_{X}$ and

$$
\eta_{i}:= \begin{cases}\mathrm{Id}: Y_{0} \rightarrow X_{0} & \text { if } i=0 \\ \gamma_{i} \circ \cdots \circ \gamma_{1}: Y_{i} \rightarrow X_{i} & \text { if } i>0 .\end{cases}
$$

The spaces and morphisms form a diagram as in (2.1.1) that satisfies all assumptions made in Theorem 2.1. Theorem 1.1 follows.

\section{DiRECT APPLICATIONS}

We will prove Theorems 1.5, 1.9, 1.10 and 1.13 in this section.

7.1. Proof of Theorem 1.5. Let $X$ be any variety that satisfies the assumptions of Theorem 1.5. To prove the theorem, we will first show that there exists a quasiétale Galois cover $\widetilde{X} \rightarrow X$ that satisfies Statement (1.5.1). The equivalence between (1.5.1) and (1.5.2) is then shown separately.

Step 1: Proof of Statement (1.5.1). Aiming for a contradiction, we assume that given any normal, quasi-projective variety $\widetilde{X}$ and any quasi-étale Galois morphism $\gamma: \widetilde{X} \rightarrow X$, there exists a normal, quasi-projective variety $\widehat{X}^{\circ}$ and an étale cover $\psi^{\circ}: \widehat{X}^{\circ} \rightarrow \widetilde{X}_{\text {reg }}$ that is not the restriction of any étale cover of $\widetilde{X}$.

Given any $\widetilde{X}$ as above, Theorem 3.8 asserts that any étale cover of $\widetilde{X}_{\text {reg }}$ extends to a cover of $\widetilde{X}$. The assumption is therefore equivalent to the following.

Assumption 7.1. Given any normal, quasi-projective variety $\widetilde{X}$ and any quasi-étale, Galois morphism $\gamma: \widetilde{X} \rightarrow X$, there exists a normal, quasi-projective variety $\widehat{X}$ and a cover $\psi: \widehat{X} \rightarrow \widetilde{X}$ that is étale over $\widetilde{X}_{\text {reg, but not étale. }}$

Using Assumption 7.1 repeatedly, and taking Galois closures as in Theorem 3.7, one inductively constructs a sequence of covers,

$$
X=Y_{0} \stackrel{\gamma_{1}}{\longleftarrow} Y_{1} \stackrel{\gamma_{2}}{\longleftarrow} Y_{2} \stackrel{\gamma_{3}}{\longleftarrow} Y_{3} \stackrel{\gamma_{4}}{\longleftarrow} \cdots,
$$

where all $\gamma_{i}$ are quasi-étale, but not étale and where all composed morphisms $\gamma_{1} \circ \cdots \circ \gamma_{i}$ are Galois. We obtain a contradiction to Theorem 1.1, showing our initial assumption was absurd. This finishes the proof of Statement (1.5.1).

Step 2: Proof of Implication (1.5.1) $\Rightarrow$ (1.5.2). If $\widetilde{X} \rightarrow X$ is any cover for which Statement (1.5.1) holds, we claim that the push-forward map $\widehat{\iota}_{*}: \widehat{\pi}_{1}\left(\widetilde{X}_{\text {reg }}\right) \rightarrow$ $\widehat{\pi}_{1}(\widetilde{X})$ of étale fundamental groups is isomorphic. For surjectivity, recall from [FL81, 0.7.B on p. 33] and [Ko195, Prop. 2.10] that the push-forward map $\iota_{*}$ : $\pi_{1}\left(\widetilde{X}_{\text {reg }}^{a n}\right) \rightarrow \pi_{1}\left(\widetilde{X}^{a n}\right)$ between topological fundamental groups is surjective. Because profinite completion is a right-exact functor, [RZ10, Lem. 3.2.3 and Prop. 3.2.5], it follows that $\widehat{\iota}_{*}$ is likewise surjective.

To show that $\widehat{\iota}_{*}$ is injective, we use Grothendieck's equivalence between the category of étale covers and the category of finite sets with transitive action of the étale fundamental group, [Mil80, Sect. 5]. Arguing by contradiction, assume that there exists a non-trivial element $g \in \operatorname{ker} \widehat{\iota}_{*}$. Since $\widehat{\pi}_{1}\left(\widetilde{X}_{\text {reg }}\right)$ is the profinite completion of $\pi_{1}\left(\widetilde{X}_{\text {reg }}^{a n}\right)$, it is residually finite. Hence, there exists a finite group $H$ and a surjective group homomorphism $\eta: \widehat{\pi}_{1}\left(\widetilde{X}_{\text {reg }}\right) \rightarrow H$ such that $\eta(g) \neq 0$. By choice 
of $g$, the natural action of $\widehat{\pi}_{1}\left(\widetilde{X}_{\mathrm{reg}}\right)$ on $H$ is not induced by an action of $\widehat{\pi}_{1}(\widetilde{X})$. Using Grothendieck's equivalence, we obtain an associated étale cover of $\widetilde{X}_{\text {reg }}$ that is not the restriction of any étale cover of $\widetilde{X}$. This contradiction to Statement (1.5.1) shows that $\widehat{\iota}_{*}$ is injective and finishes the proof of Statement (1.5.2).

Step 3: Proof of Implication (1.5.2) $\Rightarrow$ (1.5.1). This is immediate from Grothendieck's equivalence. The proof of Theorem 1.5 is thus finished.

7.1.1. Further remarks. In the setting of Theorem 1.5 , the set $\widetilde{U}:=\gamma^{-1}\left(X_{\mathrm{reg}}\right)$ is a big open subset of the smooth variety $\widetilde{X}_{\text {reg. }}$. The topological fundamental groups of the complex manifolds $\widetilde{U}$ and $\widetilde{X}_{\text {reg }}^{a n}$ therefore agree, and the inclusions $\widetilde{U} \subseteq \widetilde{X}_{\text {reg }} \subseteq \widetilde{X}$ induce a sequence of isomorphism between étale fundamental groups

$$
\widehat{\pi}_{1}(\widetilde{U}) \stackrel{\text { isomorphism }}{\longrightarrow} \widehat{\pi}_{1}\left(\widetilde{X}_{\text {reg }}\right) \stackrel{\text { isomorphism }}{\longrightarrow} \widehat{\pi}_{1}(\widetilde{X}) .
$$

7.2. Proof of Theorem 1.9. The proof of Theorem 1.9 follows the argumentation of Section 7.1 quite closely, using Theorem 2.1 instead of the simpler Theorem 1.1. Maintaining notation and assumptions of Theorem 1.9, we argue by contradiction and assume the following.

Assumption 7.2. For any open neighbourhood $X^{\circ}$ of $p$ in $X$ and any quasi-étale, Galois morphism $\gamma: \widetilde{X}^{\circ} \rightarrow X^{\circ}$, there exists an open neighbourhood $U=U(p) \subseteq$ $X^{\circ}$ with preimage $\widetilde{U}=\gamma^{-1}(U)$, and coverings

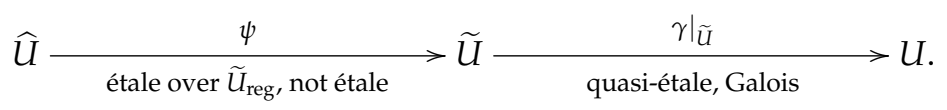

Using the existence of a Galois closure and the invariance of the branch locus, Theorem 3.7 and (3.7.2), we are free to assume the following in addition.

Assumption 7.3. The composed morphism $\left.\gamma\right|_{\widetilde{U}} ^{\circ} \psi$ is Galois.

Using Assumption 7.2, we will inductively construct an infinite diagram of morphisms as in Theorem 2.1,

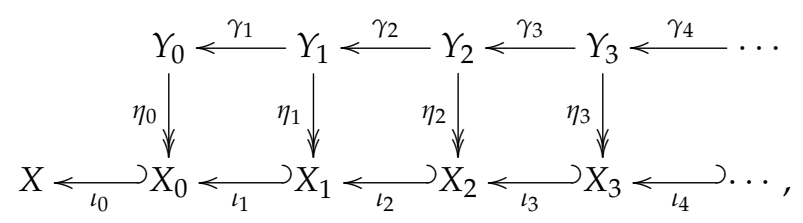

where all $\gamma_{i}$ are quasi-étale, but not étale. This will lead to a contradiction when we apply Theorem 2.1 with $S=X_{\text {sing }}$.

Construction 7.5 (Construction up to $\eta_{1}$ ). Applying Assumption 7.2 with $X^{\circ}:=X$ and $\gamma:=\operatorname{Id}_{X}$, obtain an open neighbourhood $U=U(p) \subseteq X^{\circ}$ and a covering $\psi$ as in (7.2.1). Set

$$
\begin{array}{llll}
X_{0}:=U & X_{1}:=U & Y_{0}:=\widetilde{U} & Y_{1}:=\widehat{U} \\
\eta_{0}:=\left.\gamma\right|_{\widetilde{U}} & \eta_{1}:=\left.\gamma\right|_{\widetilde{U}} \circ \psi & \gamma_{1}:=\psi & \iota_{1}:=\operatorname{Id}_{U} .
\end{array}
$$

Observe that $\eta_{0}$ and $\eta_{1}$ are quasi-étale. The morphism $\gamma_{1}$ is quasi-étale, but not étale. Assumption 7.3 guarantees that $\eta_{0}$ and $\eta_{1}$ are Galois.

Construction 7.6 (Construction of of $\eta_{i+1}$ ). Assume that a diagram as in (7.4.1) has been constructed, up to $\eta_{i}$. Applying Assumptions 7.2 and 7.3 with $X^{\circ}:=X_{i}$ and 
$\gamma:=\eta_{i}$, we obtain an open neighbourhood $U=U(p) \subseteq X_{i}$ and a covering $\psi$ as in (7.2.1). Set

$$
X_{i+1}:=U \quad Y_{i+1}:=\widehat{U} \quad \gamma_{i+1}:=\psi \quad \eta_{i+1}:=\left.\eta_{i}\right|_{\widetilde{U}^{\circ}} \psi
$$

Observe that $\eta_{i+1}$ is quasi-étale. The morphism $\gamma_{i+1}$ is quasi-étale, but not étale. Assumption 7.3 guarantees that $\eta_{i+1}$ is Galois.

In summary, we have obtained a contradiction to Theorem 2.1 showing that Assumption 7.2 was absurd. This finishes the proof of Theorem 1.9.

7.3. Proof of Theorem 1.10. Before starting with the proof of Theorem 1.10 we note the following elementary fact which will be used throughout.

Fact 7.7. Let $X$ be a quasi-projective variety, $S \subset X$ any finite set and $\mathscr{L}$ any invertible sheaf on $X$. Then there exists a Zariski-open set $U \subseteq X$ that contains $S$ and trivialises $\mathscr{L}$, that is, $\left.\mathscr{L}\right|_{U} \cong \mathscr{O}_{U}$.

Step 1: Proof of Statement (1.10.1). Maintaining notation and assumptions of Theorem 1.10 and using the assertions of Theorem 1.9, let $U \subseteq X^{\circ}$ be any Zariskiopen neighbourhood of $p$ and $\widetilde{D}$ be any Q-Cartier Weil divisor on $\widetilde{U}$. Given any point $x \in \widetilde{U}$, we need to show that $\widetilde{D}$ is Cartier at $x$.

We claim that there exists a Zariski-open subset $V \subseteq U$ that contains $p$ and $\gamma(x)$, and a number $n$ such that $\mathscr{O}_{\widetilde{V}}(n \cdot D) \cong \mathscr{O}_{\widetilde{V}}$, where $\widetilde{V}=\gamma^{-1}(V)$. In order to construct $V$, consider the open, Galois-invariant set $S:=\gamma^{-1}(p) \cup \gamma^{-1}(\gamma(x))$. By Fact 7.7, there exists an open subset $\widetilde{W} \subseteq \widetilde{U}$ that contains $S$ and a number $n$ such that $\mathscr{O}_{\widetilde{W}}(n \cdot D) \cong \mathscr{O}_{\widetilde{W}}$. Set $\widetilde{V}:=\bigcap_{g \in \operatorname{Gal}(\gamma)} g^{-1}(\widetilde{W})$. This is an open subset of $\widetilde{W}$ that contains $S$, satisfies $\mathscr{O}_{\widetilde{V}}(n \cdot D) \cong \mathscr{O}_{\widetilde{V}}$ and is invariant under the action of the Galois group. The last point implies that $\widetilde{V}$ is of the form $\gamma^{-1}(V)$, for an open subset $V \subseteq U$ that contains $p$ and $\gamma(x)$.

Continuing the proof of Statement (1.10.1), choose $n$ minimal, and let $\eta: \widehat{V} \rightarrow \widetilde{V}$ be the associated index-one cover. The covering map $\eta$ is quasi-étale and branches exactly over those points of $\widetilde{V}$ where $\widetilde{D}$ fails to be Cartier. In particular, its restriction

$$
\eta^{\circ}:=\left.\eta\right|_{\eta^{-1}\left(\widetilde{V}_{\text {reg }}\right)}: \eta^{-1}\left(\widetilde{V}_{\text {reg }}\right) \rightarrow \widetilde{V}_{\text {reg }}
$$

is étale. Recall from Theorem 1.9 that $\eta^{\circ}$ admits an étale extension to all of $\widetilde{V}$. The uniqueness assertion in Zariski's Main Theorem, Theorem 3.8, therefore implies that this extension equals $\eta$, so that $\eta$ is itself étale. As $x \in \widetilde{V}$, this finishes the proof of Statement (1.10.1).

Step 2: Proof of Statement (1.10.2). For simplicity of notation, write $G=\operatorname{Gal}(\gamma)$ and let $m$ denote the size of this group. Given any open neighbourhood $U=$ $U(p) \subseteq X^{\circ}$, any Q-Cartier divisor $D$ on $U$ and any point $x \in U$, we need to show that $m \cdot D$ is Cartier at $x$. Consider the pull-back $\widetilde{D}:=\gamma^{*} D$ and recall from Statement (1.10.1) that $\widetilde{D}$ is Cartier on $\widetilde{U}$. Again using Fact 7.7, find an open neighbourhood $V$ of $x$ with preimage $\widetilde{V}:=\gamma^{-1}(V)$ such that $\left.\widetilde{D}\right|_{\widetilde{V}}$ is linearly equivalent to zero. In other words, $\left.\widetilde{D}\right|_{\widetilde{V}}=\operatorname{div}(\widetilde{f})$, where $\widetilde{f}$ is a suitable rational function on $\widetilde{V}$. Taking averages, we obtain a rational function $\widetilde{F}:=\prod_{g \in G} \widetilde{f} \circ g$, the norm of $\widetilde{f}$, which is Galois invariant, therefore descends to a rational function $F$ on $V$, and defines the divisor $\operatorname{div} \widetilde{F}=m \cdot \widetilde{D}$. To finish the proof of Statement (1.10.2), observe that $\operatorname{div} F=m \cdot D$. This also finishes the proof of Theorem 1.10. 
7.4. Proof of Theorem 1.13. We prove Theorem 1.13 as an application of the following, more general Proposition 7.8, which we expect to have further applications, for example in the classification theory of singular varieties with trivial canonical class; cf. [GKP11, Sect. 8.C].

Proposition 7.8. Let $\mathcal{R}$ be a set of normal, quasi-projective varieties satisfying the following conditions.

(7.8.1) If $X \in \mathcal{R}$ and if $Y \rightarrow X$ is any quasi-étale Galois cover, then $Y \in \mathcal{R}$.

(7.8.2) For each $X \in \mathcal{R}$, there exists a $\mathbb{Q}$-Weil divisor $\Delta$ such that $(X, \Delta)$ is klt.

(7.8.3) Each variety $X \in \mathcal{R}$ has finite étale fundamental group.

Then, for each $X \in \mathcal{R}$, the étale fundamental group $\widehat{\pi}_{1}\left(X_{\text {reg }}\right)$ of $X_{\text {reg }}$ is finite.

Proof. Let $X \in \mathcal{R}$. By assumption (7.8.2), there exists a Q-Weil divisor $\Delta$ such that the pair $(X, \Delta)$ is klt. Let $\gamma: \widetilde{X} \rightarrow X$ be a Galois cover with the properties listed in Theorem 1.5. In particular, $\widehat{\pi}_{1}\left(\widetilde{X}_{\text {reg }}\right) \simeq \widehat{\pi}_{1}(\widetilde{X})$. We obtain an exact sequence as follows,

$$
1 \rightarrow \pi_{1}\left(\gamma^{-1}\left(X_{\text {reg }}^{a n}\right)\right) \rightarrow \pi_{1}\left(X_{\text {reg }}^{a n}\right) \rightarrow \operatorname{Gal}(\gamma) \rightarrow 1
$$

Recalling from Section 7.1.1 that $\pi_{1}\left(\gamma^{-1}\left(X_{\text {reg }}^{a n}\right)\right)=\pi_{1}\left(\widetilde{X}_{\text {reg }}^{a n}\right)$ and using the rightexactness of the profinite completion functor, [RZ10, Lem. 3.2.3 and Prop. 3.2.5], we obtain the following exact sequence of profinite completions,

$$
\widehat{\pi}_{1}(\widetilde{X}) \rightarrow \widehat{\pi}_{1}\left(X_{\text {reg }}\right) \rightarrow \operatorname{Gal}(\gamma) \rightarrow 1 \text {. }
$$

By Assumption (7.8.1), the variety $\widetilde{X}$ is in $\mathcal{R}$, and $\widehat{\pi}_{1}(\widetilde{X})$ is hence finite. Consequently, Sequence (7.8.4) presents $\widehat{\pi}_{1}\left(X_{\text {reg }}\right)$ as an extension of two finite groups, which concludes the proof.

Proof of Theorem 1.13. For the purpose of this proof, a normal projective variety $X$ is called weakly Fano if there exists a $Q$-Weil divisor $\Delta$ such that the pair $(X, \Delta)$ is klt, and $-\left(K_{X}+\Delta\right)$ is nef and big. We claim that $\mathcal{R}=$ \{weakly Fano varieties satisfies the assumptions of Proposition 7.8. We note that (7.8.2) is trivially satisfied, and that (7.8.3) follows from a result of Takayama [Tak00, Thm. 1.1]; see also [Zha06, Cor. 1], which asserts that any weakly Fano variety is simply connected.

In order to show the remaining Property (7.8.1), we let $X$ be weakly Fano with boundary divisor $\Delta$, and $\gamma: Y \rightarrow X$ be a quasi-étale cover. Use finiteness of $\gamma$ to define a pull-back, $\Delta_{Y}:=\gamma^{*}(\Delta)$, and use finiteness of $\gamma$ as well as [KM98, Prop. 5.20] to conclude that the pair $\left(Y, \Delta_{Y}\right)$ is klt. In fact, more is true. Since $\gamma$ is quasi-étale, there exists a Q-linear equivalence $K_{Y}+\Delta_{Y} \sim_{Q} \gamma^{*}\left(K_{X}+\Delta\right)$, which shows that $-\left(K_{Y}+\Delta_{Y}\right)$ is nef and big. In other words, $Y$ is weakly Fano.

\section{FLAT SHEAVES ON KLT BASE SPACES}

8.1. Proof of Theorem 1.14. Recalling the set-up of Theorem 1.14, let $X$ be a normal, complex, quasi-projective variety and assume that there exists a Q-Weil divisor $\Delta$ such that $(X, \Delta)$ is klt. We will prove that there exists a finite, surjective Galois morphism $\gamma: \widetilde{X} \rightarrow X$, étale in codimension one, such that for any locally free, flat, analytic sheaf $\mathscr{G}^{\circ}$ on $\widetilde{X}_{\text {reg, }}^{a n}$, there exists a locally free, flat, analytic sheaf $\mathscr{G}^{a n}$ on $\widetilde{X}^{a n}$ such that $\left.\mathscr{G}^{a n}\right|_{X_{\mathrm{reg}}^{a n}} \cong \mathscr{G}^{\circ}$. Recalling from [Del70, II.5, Cor. 5.8 and Thm. 5.9], that there exists a coherent, reflexive, algebraic sheaf $\mathscr{G}$ on $\widetilde{X}$ whose analytification over $\widetilde{X}_{\text {reg }}$ equals $\mathscr{G}^{\circ}$, the claim will then follow; cf. [Gro70].

Let $\widetilde{X} \rightarrow X$ be any cover for which the assertions of Theorem 1.5 hold true. To shorten notation, we denote the relevant complex spaces by $Y:=\widetilde{X}^{a n}$ and $Y^{\circ}:=$ $\widetilde{X}_{\text {reg. }}^{a n}$. The inclusion is denoted by $\iota: Y^{\circ} \rightarrow Y$. We have seen in Statement (1.5.2) 
and Section 7.1.1 that the induced morphism of étale fundamental groups, $\widehat{\iota}_{*}$ : $\widehat{\pi}_{1}\left(Y^{\circ}\right) \rightarrow \widehat{\pi}_{1}(Y)$, is isomorphic.

By Definition 1.15, the sheaf $\mathscr{G}^{\circ}$ corresponds to a representation $\rho^{\circ}: \pi_{1}\left(Y^{\circ}\right) \rightarrow$ $\operatorname{GL}(\operatorname{rank} \mathscr{F}, \mathbb{C})$. We write $G:=\operatorname{img}\left(\rho^{\circ}\right)$. The group $G$ is a quotient of the finitely generated group $\pi_{1}\left(Y^{\circ}\right)$, hence finitely generated. As a subgroup of the general linear group, $G$ is residually finite by Malcev's theorem, [Weh73, Thm. 4.2]. Consequently, the profinite completion morphism $a: G \rightarrow \widehat{G}$ is injective, [RZ10, Sect. 3.2].

To give an extension of $\mathscr{G}^{\circ}$ to a flat sheaf on $Y$, we need to show that the representation $\rho^{\circ}$ is the restriction of a representation $\rho$ of $\pi_{1}(Y)$. More precisely, we need to find a factorisation

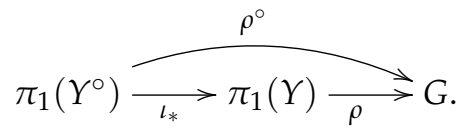

To this end, recall from [RZ10, Lem. 3.2.3] that taking profinite completion is functorial. Hence, we obtain a commutative diagram,

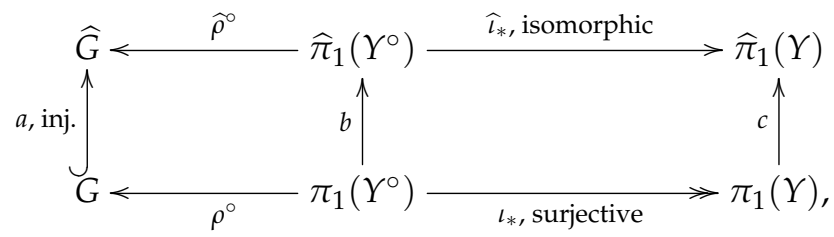

where all vertical arrows are the natural profinite completion morphisms. Since $\widehat{\iota}_{*}$ is isomorphic by construction, we can set $\rho:=\widehat{\rho}^{\circ} \circ\left(\widehat{\iota}_{*}\right)^{-1} \circ c$. Recalling from [Kol95, Prop. 2.10] that $l_{*}$ is surjective, it follows from commutativity that $\operatorname{img}(\rho) \subseteq \operatorname{img}(a)$. Identifying $G$ with its image under $a$, we have thus constructed a factorisation as in (8.0.5). This finishes the proof of Theorem 1.14.

8.2. Proof of Corollary 1.16. Let $\gamma: \widetilde{X} \rightarrow X$ be any cover for which the assertion of Theorem 1.14 holds true. By assumption, $\gamma$ is étale in codimension one, hence

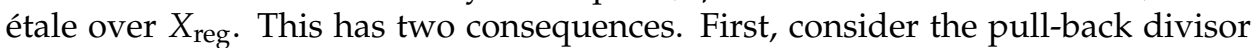
$\Delta_{\widetilde{X}}:=\gamma^{*}(\Delta)$. The pair $\left(\widetilde{X}, \Delta_{\widetilde{X}}\right)$ is then klt, [KM98, Prop. 5.20]. Second, it follows that $\mathscr{T}_{\widetilde{X}^{\circ}} \cong \gamma^{*}\left(\mathscr{T}_{\mathrm{reg}}\right)$ is a locally free, flat sheaf on $\widetilde{X}^{\circ}:=\gamma^{-1}\left(X_{\text {reg }}\right)$. Hence, $\mathscr{T}_{\tilde{X}^{\circ}}$ admits an extension to a locally free, flat sheaf $\widetilde{\mathscr{F}}$ on $\widetilde{X}$. Since $\widetilde{\mathscr{F}}$ and $\mathscr{T}_{\widetilde{X}}$ agree in codimension one and since $\mathscr{T}_{\widetilde{X}}$ is reflexive, both sheaves agree on all of $\widetilde{X}$. It follows that $\mathscr{T}_{\widetilde{X}}$ is locally free and flat. In particular, $K_{\tilde{X}}$ is Cartier, $\Delta_{\tilde{X}}$ is Q-Cartier, and the pair $(\widetilde{X}, \varnothing)$ is hence klt, [KM98, Cor. 2.35]. The solution of the Lipman-Zariski conjecture for klt spaces, [GKKP11, Thm. 6.1 $]^{1}$ thus asserts that $\widetilde{X}$ is smooth. Since $\gamma$ is Galois, hence a quotient map, $X$ will automatically have quotient singularities. This proves the first statement of Corollary 1.16.

Now assume in addition that $X$ is projective. Then $\widetilde{X}$ is a smooth, projective variety with flat tangent bundle and therefore the quotient of an Abelian variety $A$ by a finite group acting freely, [Kob87, Chap. 4, Cor. 4.15]. Taking the Galois closure of the morphism $A \rightarrow X$ as in Theorem 3.7, we find a sequence of covers,

$\widetilde{\gamma}$, Galois, étale in codim. one

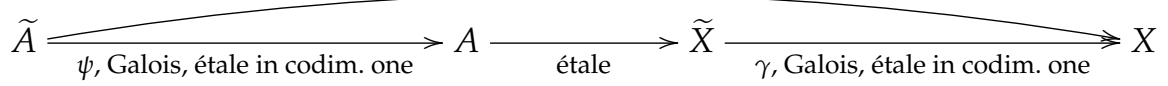

\footnotetext{
${ }^{1}$ See [Dru14, Gra15] for latest results.
} 
Since $A$ is smooth, it is clear that the morphism $\psi$, which is a priori étale only in codimension one, is in fact étale. As an étale cover of an Abelian variety, $\widetilde{A}$ is again an Abelian variety.

\section{VARIETIES WITH VANISHING CHERN CLASSES}

We prove Theorems 1.20 and 1.17 in this section. The proofs rely on a boundedness result for families of flat bundles, which we establish first.

9.1. Boundedness for families of flat bundles. We will first show that the set of flat bundles forms a bounded family. Using the results of Section 5, this will later allow to recover the isomorphism type of a bundle from its restriction to a given hyperplane.

Proposition 9.1. Let $X$ be a normal projective variety and $r \in \mathbb{N}^{+}$be any integer. Then, the set

$$
\mathrm{B}:=\{\mathscr{F} \mid \mathscr{F} \text { a locally free, flat, analytic sheaf on } X \text { with } \operatorname{rank} \mathscr{F}=r\}
$$

is a bounded family.

Proof. If $\pi: Y \rightarrow X$ is a resolution of singularities, and $E$ is a locally free, flat sheaf on $X$, then $\pi^{*} E$ is a locally free, flat, analytic sheaf on $Y$ such that $\pi_{*} \pi^{*} E \cong E$. We may therefore assume without loss of generality that $X$ is smooth. Since any locally free, flat, analytic sheaf on $X$ carries an integrable algebraic connection in the sense of [Sim94, p. 24], the claim follows from the second part of [Sim94, Thm. 6.13].

9.2. Proof of Theorem 1.20. Let $\gamma: \widetilde{X} \rightarrow X$ be a quasi-étale, Galois cover enjoying the properties stated in Theorem 1.14. Notice first that $\widetilde{X}$ is still smooth in codimension two, since $\gamma$ branches only over the singular set of $X$. Second, it follows from [KM98, Prop. 5.20] that $(\widetilde{X}, \widetilde{\Delta})$ is klt, for $\widetilde{\Delta}:=\gamma^{*} \Delta$. Since $\gamma$ is finite, the Cartier divisor $\widetilde{H}:=\gamma^{*}(H)$ is ample. It follows from [HL10, Lem. 3.2.2] that $\mathscr{F}:=\left(\gamma^{*} \mathscr{E}\right)^{* *}$ is $\widetilde{H}$-semistable. Proposition 4.6 and Assumption (1.20.1) guarantee that

$$
c_{1}(\mathscr{F}) \cdot \widetilde{H}^{n-1}=0, \quad c_{1}(\mathscr{F})^{2} \cdot \widetilde{H}^{n-2}=0, \quad \text { and } \quad c_{2}(\mathscr{F}) \cdot \widetilde{H}^{n-2}=0 .
$$

To prove Theorem 1.20, we need to show that $\mathscr{F}$ is locally free and flat.

Step 1: Construction of a representation. It follows from Proposition 9.1 that the family B of locally free, flat, coherent sheaves on $\widetilde{X}$ whose rank equals $r:=\operatorname{rank} \mathscr{F}$ is bounded. We may thus apply the Mehta-Ramanathan-Theorem for normal spaces, [Fle84, Thm. 1.2], and Corollary 5.3 to obtain an increasing sequence of numbers, $0 \ll m_{1} \ll m_{2} \ll \cdots \ll m_{n-2}$, as well as general elements $D_{i} \in\left|\mathscr{O}_{\widetilde{X}}\left(m_{i} \cdot \widetilde{H}\right)\right|$ such that the following holds.

(9.2.2) The surface $S:=D_{1} \cap \cdots \cap D_{n-2}$ is smooth and contained in $\widetilde{X}_{\text {reg. }}$.

(9.2.3) The restricted sheaf $\left.\mathscr{F}\right|_{S}$ is semistable with respect to $\left.\widetilde{H}\right|_{S}$.

(9.2.4) Let $\mathscr{B} \in \mathrm{B}$ be any member. Then, $\mathscr{F} \cong \mathscr{B}$ if and only if $\left.\left.\mathscr{F}\right|_{S} \cong \mathscr{B}\right|_{S}$. Further, it follows from (9.2.1) and Remark 4.5 that

$$
c_{1}\left(\left.\mathscr{F}\right|_{S}\right) \cdot\left(\left.\widetilde{H}\right|_{S}\right)=0 \quad \text { and } \quad \operatorname{ch}_{2}\left(\left.\mathscr{F}\right|_{S}\right)=\frac{1}{2} c_{1}\left(\left.\mathscr{F}\right|_{S}\right)^{2}-c_{2}\left(\left.\mathscr{F}\right|_{S}\right)=0,
$$

where $\mathrm{ch}_{2}$ denotes the second Chern character. With these equalities, it follows from Simpson's work, [Sim92, Cor. 3.10], that the semistable sheaf $\left.\mathscr{F}\right|_{S}$ is flat. In other words, $\left.\mathscr{F}\right|_{S}$ is given by a representation $\rho: \pi_{1}\left(S^{a n}\right) \rightarrow \operatorname{GL}(r, \mathbb{C})$. The Lefschetz Theorem for singular spaces, [GM88, Thm. in Sect. II.1.2], asserts that the natural homomorphism $\iota_{*}: \pi_{1}\left(S^{a n}\right) \rightarrow \pi_{1}\left(\widetilde{X}_{\text {reg }}^{a n}\right)$, induced by the inclusion 
$\iota: S^{a n} \hookrightarrow X_{\text {reg }}^{a n}$, is isomorphic. Composing the inverse $\left(\iota_{*}\right)^{-1}$ with $\rho$ we obtain a representation $\tau: \pi_{1}\left(\widetilde{X}_{\text {reg }}^{a n}\right) \rightarrow \mathrm{GL}(r, \mathbb{C})$.

Step 2: End of proof. The representation $\tau$ defines a flat, locally free, analytic sheaf $\mathscr{F}_{\tau}^{\circ}$ on $\widetilde{X}_{\text {reg, }}^{a n}$, which, by choice of $\widetilde{X}$, comes from a flat, locally free, algebraic sheaf $\mathscr{F}_{\tau}$ on $\widetilde{X}$. By construction, $\left.\mathscr{F}_{\tau}\right|_{S}$ is isomorphic to $\left.\mathscr{F}\right|_{S}$. Applying (9.2.4), we conclude that $\mathscr{F}$ is isomorphic to $\mathscr{F}_{\tau}$, and therefore flat. This finishes the proof of Theorem 1.20 .

9.2.1. Concluding remarks. The results of this section clearly remain true if we substitute the polarisation $(H, \ldots, H)$ by $H_{1}, \ldots, H_{n-1}$, where the $H_{j}$ are not necessarily identical ample divisors.

If $\mathscr{E}$ is polystable, it is possible to avoid the use of [Sim92, Cor. 3.10] in the proof of Theorem 1.20, by using [Don85, Thm. 1] to conclude that $\left.\mathscr{F}\right|_{S}$ is HermiteEinstein. This suffices for the proof of Theorems 9.3 and 1.17 below, since -in the setting of Theorem 1.17- the tangent sheaf is polystable for any polarisation by [GKP11, Cor. 7.3], possibly after a quasi-étale cover of $X$.

9.3. Proof of Theorem 1.17. Using the terminology introduced in Section 4 we state the main result of this section. Theorem 1.17 follows directly from this.

Theorem 9.3 (Characterisation of quotients of Abelian varieties). Let X be a normal $n$-dimensional projective variety which is klt and smooth in codimension two. Then the following conditions are equivalent:

(9.3.1) $K_{X} \equiv 0$, and $c_{2}\left(\mathscr{T}_{X}\right)$ is numerically trivial in the sense of Definition 4.7.

(9.3.2) There exists an Abelian variety $A$ and a finite, surjective, Galois morphism $A \rightarrow$ $X$ that is étale in codimension two.

Remark 9.4 (Comparing the assumptions of Theorem 9.3 and Theorem 1.17). By Proposition 4.8, the conditions in (9.3.1) are equivalent the following conditions: $K_{X} \equiv 0$, and there exist ample divisors $H_{1}, \ldots, H_{n-2}$ on $X$ such that $c_{2}\left(\mathscr{T}_{X}\right)$. $H_{1} \cdots H_{n-2}=0$. This establishes the link between Theorem 9.3 and Theorem 1.17, as stated in the introduction.

Remark 9.5. In dimension three, Theorem 9.3 has been shown without the assumption on the codimension of the singular set by Shepherd-Barron and Wilson, [SBW94], using orbifold techniques. It seems feasible to obtain an analogous result also in higher dimensions with the methods presented here.

Theorem 9.3 is shown in the remainder of this section. The two implications will be shown separately.

Proof of Theorem 9.3, (9.3.2) $\Rightarrow$ (9.3.1). If $\eta: A \rightarrow X$ is any finite map from an Abelian variety, étale in codimension two, then there exists a linear equivalence $\eta^{*}\left(K_{X}\right) \sim K_{A}=0$. In particular, it follows from the projection formula that $K_{X}$ is numerically trivial. Corollary 4.9 implies that $c_{2}\left(\mathscr{T}_{X}\right)$ is numerically trivial as well.

Proof of Theorem 9.3, (9.3.1) $\Rightarrow$ (9.3.2). Assume that (9.3.1) holds. We first reduce to the case of canonical singularities. By the abundance theorem for klt varieties with numerically trivial canonical divisor class, [Amb05, Thm. 4.2], there exists an $m \in \mathbb{N}^{+}$such that $\mathscr{O}_{X}\left(m K_{X}\right) \cong \mathscr{O}_{X}$. Let $m$ be minimal with this property, and let $v: \widehat{X} \rightarrow X$ be the associated global index-one cover, which is quasi-étale over $X$. Then, $\mathscr{O}_{\widetilde{X}}\left(K_{\tilde{X}}\right) \cong \mathscr{O}_{\widetilde{X}}$, which together with the fact that $\widetilde{X}$ has klt singularities, [KM98, Prop. 5.20], implies that $\widetilde{X}$ has canonical singularities. Moreover, applying 
Corollary 4.9 we see that $\widetilde{X}$ is smooth in codimension two, and that $c_{2}\left(\mathscr{T}_{X}\right)$ is numerically trivial. If $\eta: \widetilde{A} \rightarrow \widetilde{X}$ is a finite, surjective, Galois morphism that is étale in codimension two from an abelian variety $\widetilde{A}$ to $\widetilde{X}$, then taking the Galois closure of $v \circ \eta$ yields the desired map $A \rightarrow X$. We may therefore make the following simplifying assumption.

Assumption w.l.o.g. 9.6. The variety $X$ has canonical singularities.

Since $c_{2}(X)$ is numerically trivial, we have $c_{2}(X) \cdot H^{n-2}=0$ for any ample divisor $H$ on $X$. Since moreover $K_{X}$ is numerically trivial, and since $X$ has at worst canonical singularities by Assumption 9.6, the tangent sheaf $\mathscr{T}_{X}$ is $H$-semistable by [GKP11, Prop. 5.4], and

$$
c_{1}\left(\mathscr{T}_{X}\right) \cdot H^{n-1}=c_{1}\left(\mathscr{T}_{X}\right)^{2} \cdot H^{n-2}=0 \text { for any ample divisor } H \text { on } X .
$$

By Theorem 1.20, there hence exists a quasi-étale cover $\gamma: \widetilde{X} \rightarrow X$ such that $\mathscr{T}_{\widetilde{X}} \cong\left(\gamma^{*} \mathscr{T}_{X}\right)^{* *}$ is a flat, locally free sheaf. Applying Corollary 1.16 to $\widetilde{X}$ and possibly taking Galois closure finishes the proof of Theorem 9.3.

\section{VARIETIES ADMITTING POLARISED ENDOMORPHISMS}

We will prove Theorem 1.21 in Section 10.1. As noted in the introduction, Theorem 1.21 has consequences for the structure theory of varieties with endomorphisms. These are discussed in Section 10.2 below.

10.1. Proof of Theorem 1.21. Theorem 1.21 has been shown in [NZ10, Thm. 3.3] under an additional assumption concerning fundamental groups of smooth loci of Euclidean-open subsets of $X$. Nakayama and Zhang use this assumption only once, to prove a claim which appears on Page 1004 of their paper. After briefly recalling their setup, we will show that the claim follows directly from our Theorem 1.1, without any additional assumption. Once this is done, the original proof of Nakayama-Zhang applies verbatim.

Under the assumptions of Theorem 1.21, let $f: X \rightarrow X$ be a polarised endomorphism. Nakayama-Zhang start the proof of [NZ10, Thm. 3.3] on page 1004 of their paper by recalling from [NZ10, Thm. 3.2] that $X$ has at worst canonical singularities, that $K_{X}$ is Q-linearly trivial, and that $f$ is étale in codimension one. Given any index $k \in \mathbb{N}^{+}$, they consider the iterated endomorphism $f^{k}$ and its Galois closure,

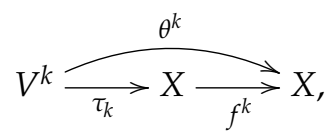

where $\theta_{k}$ and $\tau_{k}$ are Galois and again étale in codimension one; cf. Theorem 3.7. By [NZ10, Lem. 2.5], there exist finite morphisms $g_{k}, h_{k}$, again étale in codimension one, forming commutative diagrams as follows,

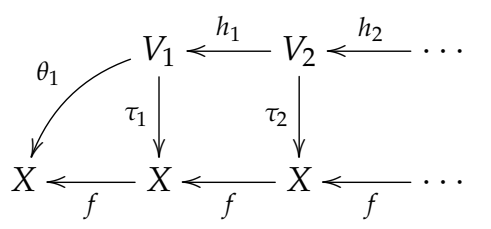

and

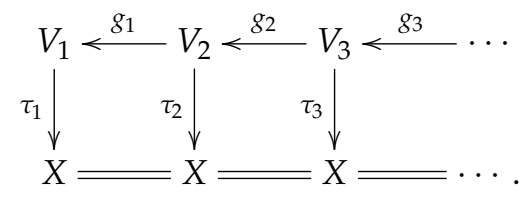

Nakayama-Zhang claim in [NZ10, claim on p. 1004] that the morphisms $h_{k}$ and $g_{k}$ are étale for all sufficiently large $k$. They prove this claim using their additional 
assumption on the fundamental groups. Theorem 1.1, however, applies to the associated sequences,

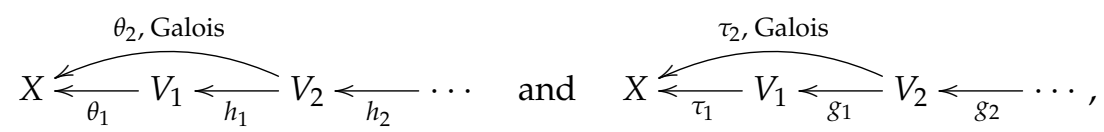

and yields this result without any extra assumption. As pointed out above, the rest of Nakayama-Zhang's proof applies verbatim.

10.2. The structure of varieties admitting endomorphisms. Theorem 1.21 has consequences for the structure theory of varieties with endomorphisms. The following results have been shown in [NZ10, Thm. 1.3], conditional to the assumption that [NZ10, Conj. 1.2] = Theorem 1.21 holds true. The definition $q^{\sharp}$ is recalled in Remark 10.2 below.

Theorem 10.1 (Structure of varieties admitting polarized endomorphisms). Let $f$ : $X \rightarrow X$ be a non-isomorphic, polarised endomorphism of a normal, complex, projective variety $X$ of dimension $n$. Then $\kappa(X) \leq 0$ and $q^{\sharp}(X, f) \leq n$. Furthermore, there exists an Abelian variety $A$ of dimension $\operatorname{dim} A=q^{\sharp}(X, f)$ and a commutative diagram of normal, projective varieties,

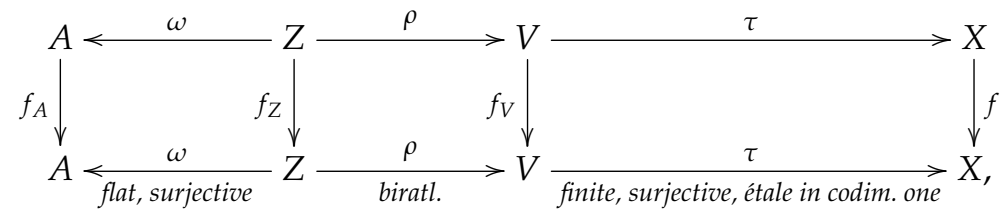

where all vertical arrows are polarised endomorphism, and every fibre of $\omega$ is irreducible, normal and rationally connected. In particular, $X$ is rationally connected if $q^{\sharp}(X, f)=0$.

Moreover, the fundamental group $\pi_{1}(X)$ contains a finitely generated, Abelian subgroup of finite index whose rank is at most $2 \cdot q^{\sharp}(X, f)$.

Remark 10.2 (Definition of $q^{\sharp}$, [NZ10, p. 992f]). In the setting of Theorem 10.1, the number $q^{\sharp}(X, f)$ is defined as the supremum of irregularities $q\left(\widetilde{X}^{\prime}\right)=h^{1}\left(\widetilde{X}^{\prime}, \mathscr{O}_{\widetilde{X}^{\prime}}\right)$ of a smooth model $\widetilde{X}^{\prime}$ of $X^{\prime}$ for all the finite coverings $\tau: X^{\prime} \rightarrow X$ étale in codimension one and admitting an endomorphism $f^{\prime}: X^{\prime} \rightarrow X^{\prime}$ with $\tau \circ f^{\prime}=f \circ \tau$.

\section{EXAMPLES, COUNTEREXAMPLES, AND SHARPNESS OF RESULTS}

In this section, we have collected several examples which illustrate to what extend our main results are sharp. In Section 11.1 we construct an infinite sequence of branched non-Galois coverings of the singular Kummer surface, showing that Theorem 1.1 does not to hold without the Galois assumption. Section 11.2 discusses an example of Gurjar-Zhang, showing that Theorem 1.13 is sharp, and that Theorem 1.1 has no simple reformulation in terms of the push-forward between fundamental groups. Finally, Section 11.3 shows by way of an example that there is generally no canonical, minimal choice for the coverings constructed in Theorem 1.5.

11.1. Isogenies of Abelian surfaces and the Kummer construction. We first construct a number of special endomorphisms of Kummer surfaces. For this, fix one Abelian surface $A$ throughout this section.

Construction 11.1 (Endomorphisms of singular Kummer surfaces). Consider the involutive automorphism $\sigma: A \rightarrow A, a \mapsto-a$. Set $X:=A / \sigma$ and denote the quotient morphism by $f: A \rightarrow X, a \mapsto[a]$. The automorphism $\sigma$ has 16 fixed 
points. The quotient surface $X$ has 16 rational double points, which are canonical and therefore klt. We call $X$ a "singular Kummer surface".

Given any number $n \in \mathbb{N}^{+}$, consider the endomorphism of $A$ obtained by multiplication with $n$, that is, $\mathbf{n}_{A}: A \rightarrow A, a \mapsto n \cdot a$. Note that $\mathbf{n}_{A}$ is a finite morphism that commutes with $\sigma$ and therefore induces a finite endomorphism of $X$, which we denote as $\mathbf{n}_{X}: X \rightarrow X,[a] \mapsto[n \cdot a]$. By construction, the morphism $\mathbf{n}_{X}$ commutes with $f$.

The main properties of the endomorphisms $\mathbf{n}_{A}$ and $\mathbf{n}_{X}$ are summarised in the following observations.

Observation 11.2 (Degree and fibres of $\mathbf{n}_{A}$ and $\mathbf{n}_{X}$ ). The morphism $\mathbf{n}_{A}$ is the quotient for the natural action of the $n$-torsion group $A_{n}<A$ on $A$. In particular, $\mathbf{n}_{A}$ is finite of degree $4^{n}$, étale and Galois. Given any point $x \in X$, it follows from commutativity, $\mathbf{n}_{X} \circ f=f \circ \mathbf{n}_{A}$, that the number of points in the fibre is at least $\# \mathbf{n}_{X}^{-1}(x) \geq \frac{1}{2} \cdot 4^{n}$.

Observation 11.3 (Endomorphism $\mathbf{n}_{X}$ is quasi-étale, not étale, and not Galois). The morphism $\mathbf{n}_{X}$ is quasi-étale by construction. Let $n>2$ be any number and $x \in$ $X_{\text {sing }}$ any singular point of $X$. Since $X$ has only 16 singularities, it follows from Observation 11.2 that the fibre $\mathbf{n}_{X}^{-1} \mathbf{n}_{X}(x)$ contains both singular and non-singular points of $X$. This shows that $\mathbf{n}_{X}$ is neither étale nor Galois if $n>2$.

Construction 11.1 shows that Theorem 1.1 does not hold without the Galois assumption. This is the content of the following proposition.

Proposition 11.4 (Sharpness of Theorem 1.1). Using the notation of Construction 11.1, choose a number $n>2$ and consider the tower of finite morphisms

$$
X \stackrel{\mathbf{n}_{X}}{\longleftarrow} X \stackrel{\mathbf{n}_{X}}{\longleftarrow} X \stackrel{\mathbf{n}_{X}}{\longleftarrow} X \stackrel{\mathbf{n}_{X}}{\longleftarrow} \cdots .
$$

Then, all assumptions of Theorem 1.1 are satisfied except that the composed morphisms $\left(\mathbf{n}^{\mathbf{k}}\right)_{X}=\mathbf{n}_{X} \circ \cdots \circ \mathbf{n}_{X}$ be Galois, for any $k>1$. However, none of the finite morphisms $\mathbf{n}_{X}$ is étale.

11.2. Comparing the étale fundamental groups of a klt variety and its smooth locus. In [GZ94, Sect. 1.15], Gurjar and De-Qi Zhang construct a rationally connected, simply-connected, projective surface $X$ with rational double point singularities, admitting a quasi-étale, two-to-one cover $\gamma: \widetilde{X} \rightarrow X$ where $\widetilde{X}$ is smooth and $\pi_{1}\left(\widetilde{X}^{a n}\right) \cong \mathbb{Z}^{2}$.

We claim that $\widehat{\pi}_{1}\left(X_{\text {reg }}\right)$ is infinite. In fact, the standard sequence

$$
0 \rightarrow \underbrace{\pi_{1}\left(\gamma^{-1}\left(X_{\text {reg }}^{a n}\right)\right)}_{\cong \pi_{1}\left(\widetilde{X}^{a n}\right)} \stackrel{\gamma_{*}}{\rightarrow} \pi_{1}\left(X_{\text {reg }}^{a n}\right) \rightarrow \mathbb{Z} / 2 \mathbb{Z} \rightarrow 0
$$

shows that the groups $n \mathbb{Z}^{2}$ are finite-index subgroups of $\pi_{1}\left(X_{\text {reg }}^{a n}\right)$, for all $n \in \mathbb{N}^{+}$. Recalling from the definition that the kernel of the profinite completion map $\pi_{1}\left(X_{\text {reg }}^{a n}\right) \rightarrow \widehat{\pi}_{1}\left(X_{\text {reg }}\right)$ equals the intersection of all finite-index subgroups, the claim follows.

The example of Gurjar-Zhang relates to our results in at least two ways.

Morphism of fundamental groups in Theorem 1.1. Given a quasi-projective, klt variety $X$, we have pointed out in Remark 1.7 that Theorem 1.1 does not assert that the kernel of the natural surjection of étale fundamental groups, $\widehat{\iota}_{*}: \widehat{\pi}_{1}\left(X_{\mathrm{reg}}\right) \rightarrow \widehat{\pi}_{1}(X)$, is finite. The example of Gurjar-Zhang proves this point. Note that the singular Kummer surface constructed in Section 11.1 above also exemplifies the same point. 
Sharpness of Theorem 1.13. It is well-known that rationally chain connected varieties have finite fundamental group, [Kol95, Thm. 4.13]. Hence, it is a natural question whether the condition " $-\left(K_{X}+\Delta\right)$ is nef and big" of Theorem 1.13 can be weakened to " $X$ rationally chain connected". The example of Gurjar-Zhang shows that this is not the case.

11.3. Non-existence of a minimal choice in Theorem 1.5. We will show in this section that, given a klt variety $X$, there is in general no canonical choice for the cover $\widetilde{X}$ constructed in Theorem 1.5 , that is minimal in the sense that any other such cover would factorise over $\widetilde{X}$. For this, we will construct a quasi-projective klt surface $X$ and two covers $\gamma_{1}: \widetilde{X}_{1} \rightarrow X, \gamma_{2}: \widetilde{X}_{2} \rightarrow X$ that satisfy the conditions of Theorem 1.5. The geometry of these covers will differ substantially. It will be clear from the construction that the two covers do not dominate a common third. In other words, there is no cover $\psi: Y \rightarrow X$ that satisfies the conclusions of Theorem 1.5 and fits into a diagram as follows,

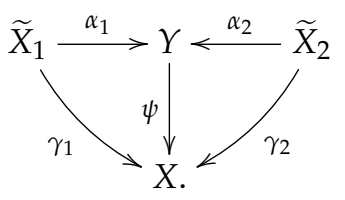

This shows that a canonical, minimal covering cannot exist.

Construction of the surface $X$. Consider the spaces $A=\mathbb{P}^{1} \times \mathbb{P}^{1}$ and $B:=\mathbb{P}^{1}$. Identifying the group $\mathbb{Z}_{2}$ with the multiplicative group $\{-1,1\}$, consider the actions of the Klein four-group $G:=\mathbb{Z}_{2} \times \mathbb{Z}_{2}$ on $A$ and $B$, written in inhomogeneous coordinates as follows

$$
\begin{aligned}
\left(\mathbb{Z}_{2} \times \mathbb{Z}_{2}\right) \times A \rightarrow A, & \left(\left(z_{1}, z_{2}\right),\left(a_{1}, a_{2}\right)\right) & \mapsto\left(z_{1} \cdot a_{1}^{z_{2}}, z_{1} z_{2} \cdot a_{2}\right) \\
\left(\mathbb{Z}_{2} \times \mathbb{Z}_{2}\right) \times B \rightarrow B, & \left(\left(z_{1}, z_{2}\right), b\right) & \mapsto\left(z_{1} \cdot b^{z_{2}}\right) .
\end{aligned}
$$

Let $\pi: A \rightarrow B$ be the projection to the first factor. This map is clearly equivariant and therefore induces a morphism between quotients,

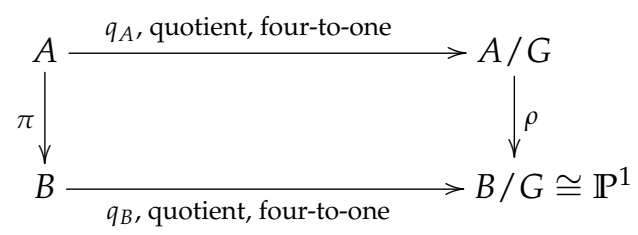

The quotient curve $B / G$ is isomorphic to $\mathbb{P}^{1}$. The quotient map $q_{B}$ has three branch points, $\left\{q_{1}, q_{2}, q_{3}\right\} \subset B / G$, and six ramification points $\{0, \infty, \pm 1, \pm i\} \subset B$, two over each of the branch points, and each with ramification index two.

The quotient surface $A / G$ is singular, with six quotient singularities of type $A_{1}$, two over each of the branch points $q_{i} \in B / G$. The quotient $A / G$ is therefore klt. Away from the branch points $q_{i}$, the morphism $\rho$ has the structure of a $\mathbb{P}^{1}$-bundle. The fibres $\rho^{-1}\left(q_{i}\right)$ are set-theoretically isomorphic to $\mathbb{P}^{1}$, but carry a non-reduced, double structure. Choose a fourth point $q_{4} \in B / G$ and consider the quasi-projective variety $X:=(A / G) \backslash \rho^{-1}\left(q_{4}\right)$. The setup is depicted in Figure 11.1.

Construction of the covering $\widetilde{X}_{1}$. Set $\widetilde{X}_{1}:=q_{A}^{-1}(X)$ and $\gamma_{1}:=\left.q_{A}\right|_{\widetilde{X}_{1}}$. Since $\widetilde{X}_{1}$ is smooth, the condition that $\widehat{\pi}_{1}\left(\widetilde{X}_{1}\right)=\widehat{\pi}_{1}\left(\left(\widetilde{X}_{1}\right)_{\text {reg }}\right)$ is satisfied. The cover $\gamma_{1}$ is Galois, four-to-one, and branches only over the singularities of $X$. 


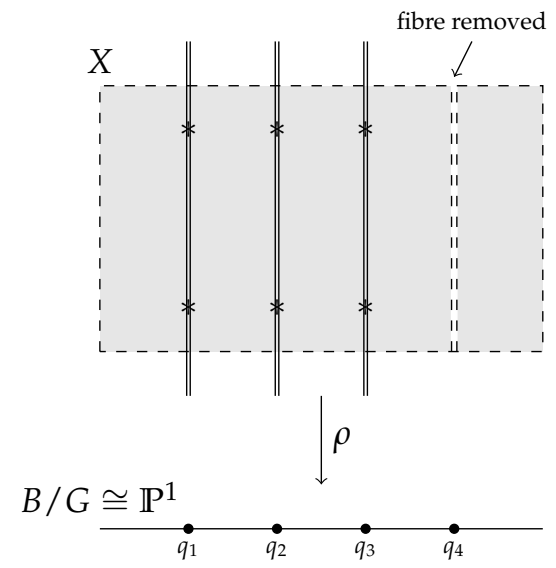

The figure shows the singular quotient surface $X$ that is constructed in Section 11.3. The surface contains six $A_{1}$ quotient singularities which are indicated with the symbol " $*$ ".

FIGURE 11.1. Singular surface with $A_{1}$ singularities

Construction of the covering $\widetilde{X}_{2}$. Consider a double covering of $B / G \cong \mathbb{P}^{1}$ by an elliptic curve $E$, branched exactly over the points $q_{1}, \ldots, q_{4}$. Let $\widetilde{X}_{2}$ be the normalisation of the fibred product $X \times_{B / G} E$ and let $\gamma_{2}: \widetilde{X}_{2} \rightarrow X$ be the obvious morphism, which is a Galois, two-to-one cover of $X$, branched exactly over the singularities of $X$. A standard computation shows that $\widetilde{X}_{2}$ is smooth. The condition that $\widehat{\pi}_{1}\left(\widetilde{X}_{2}\right)=\widehat{\pi}_{1}\left(\left(\widetilde{X}_{2}\right)_{\text {reg }}\right)$ is thus again trivially satisfied.

Non-existence of a minimal cover. We will now show that a covering $\psi: Y \rightarrow X$ forming a diagram as in (11.4.1) cannot exist. Assuming for a moment that $Y$ does exist, recall that $\gamma_{2}$ is two-to-one. It follows that either $\alpha_{2}$ or $\psi$ is isomorphic. Neither is possible: If $\alpha_{2}$ was isomorphic, we would obtain a two-to-one covering map $\alpha_{2}^{-1} \circ \alpha_{1}: \widetilde{X}_{1} \rightarrow \widetilde{X}_{2}$. This is impossible, because $\widetilde{X}_{1}$ is rational, while $\widetilde{X}_{2}$ is not. If $\psi$ was isomorphic, then $X$ would have to satisfy the condition that $\widehat{\pi}_{1}(X)=$ $\hat{\pi}_{1}\left(X_{\text {reg }}\right)$. The existence of covering maps that branch only over the singularities shows that this is not the case.

\section{Appendices}

\section{APPENDIX A. ZARISKI'S MAIN THEOREM IN THE EQUIVARIANT SETTING}

While certainly known to experts, we were not able to find a full reference for Zariski's Main Theorem in the equivariant setting, Theorem 3.8, in the literature. The following lemma will be used.

Lemma A.1. Consider the composition of a rational map $a: V \rightarrow W_{1}$ and a finite morphism $b: W_{1} \rightarrow W_{2}$, where $V, W_{1}$ and $W_{2}$ are quasi-projective varieties. If $V$ is normal and if $b \circ a$ is a morphism, then $a$ is a morphism.

Proof. Blowing up $V$ suitably, we obtain a diagram as follows

$$
\widetilde{V} \underset{\beta_{V}, \text { blow-up }}{\stackrel{\beta_{W}, \text { morphism }}{\longrightarrow} V-\frac{-}{a}} \gg W_{1} \underset{b}{\longrightarrow} W_{2} \text {. }
$$

Since $V$ is normal, it suffices to show that given any closed point $v \in V$ with fibre $F:=\beta_{V}^{-1}(v)$, the image set $\beta_{W}(F)$ is a point. To this end, recall Zariski's 
main theorem, [Har77, III Cor. 11.4], which asserts that the fibre $F$ is connected. Consequently, so is $\beta_{W}(F)$. Using the assumption that $b \circ a$ is a morphism, observe that

$$
b\left(\beta_{W}(F)\right)=(b \circ a)\left(\beta_{V}(F)\right)=(b \circ a)(v)=\text { a point. }
$$

Since $b$ is finite, it follows that the connected set $\beta_{W}(F)$ is a point, as claimed.

A.1. Proof of Theorem 3.8. Assume that we are given a morphism $a: V^{\circ} \rightarrow W$ as in Theorem 3.8. The claims made in the theorem will be shown separately.

Step 1: Existence of a factorisation. Zariski's Main Theorem in the form of Grothendieck, [Gro66, Thm. 8.12.6], asserts the existence of a quasi-projective variety $V^{\prime}$ and a factorisation of $a$ into an open immersion $\alpha^{\prime}: V^{\circ} \rightarrow V^{\prime}$ and a finite morphism $\beta^{\prime}: V^{\prime} \rightarrow W$. Let $\eta: V \rightarrow V^{\prime}$ be the normalisation. Recalling the universal property of the normalisation map, [Har77, II Ex. 3.8], observe that there exists a morphism $\alpha: V^{\circ} \rightarrow V$ such that $\alpha^{\prime}=\alpha \circ \eta$. Finally, set $\beta:=\beta^{\prime} \circ \eta$.

Step 2: Uniqueness of the factorisation. Assume we are given two factorisations of $a$ as in Theorem 3.8, say $a=\beta_{1} \circ \alpha_{1}=\beta_{2} \circ \alpha_{2}$. We obtain a commutative diagram of morphisms and rational maps,

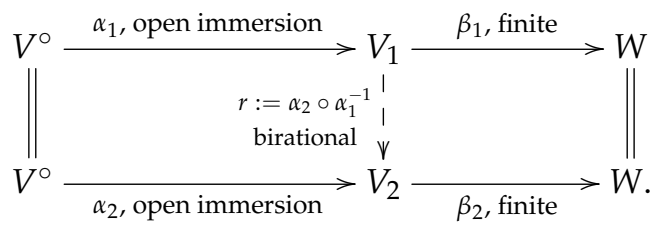

Since the composed morphism $\beta_{2} \circ r$ equals $\beta_{1}$, Lemma A.1 asserts that $r$ is a morphism. The same holds for $r^{-1}=\alpha_{1} \circ \alpha_{2}^{-1}$, showing that $r$ is isomorphic.

Step 3: The $G$-action on $V$. To show that $G$ acts on $V$, let $g \in G$ be any element. The action of $g$ gives rise to a diagram

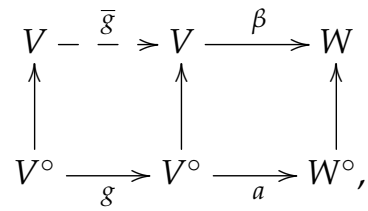

where all vertical arrows are the natural inclusion maps and where $\bar{g}$ is the natural extension of $g$ to a birational endomorphism of $V$. The morphism $a$ is $G$-invariant, which means that $a \circ g=g \circ a$. As a consequence, we see that $\beta \circ \bar{g}=g \circ \beta$. In particular, the rational map $\beta \circ \bar{g}$ is a morphism. Lemma A.1 thus implies that $\bar{g}$ is a morphism, showing that $G$ acts on $V$, as claimed. Equivariance of $\alpha$ and $\beta$ is now automatic.

Step 4: Quotients. Under the assumptions of (3.8.2), we have seen that $G$ acts on $V$. We need to show that $W$ is the quotient of the $G$-action on $V$ and that $\beta$ is the quotient map. To this end, consider the following natural diagram of (normal) quotient spaces and quotient maps,

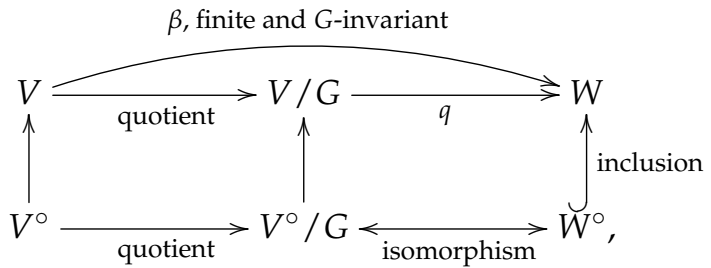


where all vertical arrows are the natural inclusion maps. The map $q$ is induced by the universal property of the quotient, [Mum08, Thm. 1 on p. 111], using that the map $\beta$ is G-invariant. The isomorphism between $V^{\circ} / G$ and $W^{\circ}$ shows that $q$ is birational.

Since $\beta$ is finite, so is $q$. The standard version of Zariski's Main Theorem, [Har77, V Thm. 5.2], therefore applies. It asserts that $V / G$ is isomorphic to $W$ and that $\beta$ is the quotient map. This finishes the proof of Theorem 3.8.

\section{APPENDix B. GALOIS CLOSURE}

B.1. Proof of Theorem 3.7. We will use the equivalence between the category of connected, étale covers of a variety $V$ and the category of finite sets $S$ with transitive action of the étale fundamental group $\widehat{\pi}_{1}(V)$, [Mil80, Sect. 5 and Thm. 5.3].

Step 1: Construction of $\widetilde{X}$. Consider the maximal open set $Y^{\circ}:=Y \backslash \operatorname{Branch}(\gamma)$ where $\gamma$ is étale. Let $X^{\circ}:=\gamma^{-1}\left(Y^{\circ}\right)$ denote the preimage. Choose a closed point $y \in Y^{\circ}$ and a closed point $x$ of the fibre $X_{y}:=\gamma^{-1}(y)$. The étale map $\left.\gamma\right|_{X^{\circ}}$ : $X^{\circ} \rightarrow Y^{\circ}$ corresponds to a transitive action of the étale fundamental group $\widehat{\pi}:=$ $\widehat{\pi}_{1}\left(Y^{\circ}, y\right)$ on $X_{y}$. The group $\hat{\pi}$ also acts on

$$
G:=\operatorname{img}\left(\widehat{\pi} \rightarrow \text { Permutation group of } X_{y}\right)
$$

by left multiplication. We obtain a sequence of finite sets with transitive $\widehat{\pi}$-action and a corresponding sequence of étale covers,

$$
G \stackrel{g \mapsto g \cdot x}{\longrightarrow} X_{y} \stackrel{\text { const. }}{\longrightarrow}\{y\} \quad \text { and } \quad \widetilde{X}^{\circ} \stackrel{\tilde{\gamma}^{\circ}}{\longrightarrow} X^{\circ} \stackrel{\left.\gamma\right|_{X^{\circ}}}{\longrightarrow} Y^{\circ},
$$

where $\Gamma^{\circ}:=\left(\left.\gamma\right|_{X^{\circ}}\right) \circ \widetilde{\gamma}^{\circ}$ is Galois with group $G$ and $\widetilde{\gamma}^{\circ}$ is Galois with Galois group equal to the isotropy group $H:=$ Iso $_{x} \leqslant G$ of the point $x \in X_{y}$, respectively. Applying Theorem 3.8 to the quasi-finite morphism $\widetilde{X}^{\circ} \rightarrow X$, we obtain a normal, quasi-projective variety $\widetilde{X}$ and a diagram

$$
\widetilde{X}^{\circ} \stackrel{\text { immersion }}{\longrightarrow} \widetilde{X} \stackrel{\widetilde{\gamma} \text {, Galois with group } H}{\longrightarrow} X \stackrel{\gamma \text {, finite }}{\longrightarrow} Y .
$$

Step 2: Verification of (3.7.1). We need to show that $\Gamma:=\gamma \circ \widetilde{\gamma}$ is Galois with group G. Applying Theorem 3.8 to the quasi-finite morphism $\widetilde{X}^{\circ} \rightarrow Y$, we obtain a normal, quasi-projective variety $\widetilde{X}^{\prime}$ and morphisms $\widetilde{X}^{\circ} \hookrightarrow \widetilde{X}^{\prime} \stackrel{\Gamma^{\prime}}{\rightarrow} Y$, where $\Gamma^{\prime}$ is Galois with group $G$. Since $\Gamma$ and $\Gamma^{\prime}$ are both finite morphisms, the uniqueness statement of Theorem 3.8 shows that $\widetilde{X}=\widetilde{X}^{\prime}$ and $\Gamma=\Gamma^{\prime}$. This establishes (3.7.1).

Step 3: Verification of (3.7.2). As $\Gamma=\gamma \circ \widetilde{\gamma}$, it follows from [Gro71, I. Thm. 10.11] that $\operatorname{Branch}(\Gamma) \supseteq \operatorname{Branch}(\gamma)$. On the other hand, the construction immediately shows that $\left(\gamma \mid X^{\circ}\right) \circ \widetilde{\gamma}^{\circ}$ is étale, so that $\operatorname{Branch}(\Gamma) \subseteq \operatorname{Branch}(\gamma)$. The branch loci of $\gamma$ and $\Gamma$ therefore coincide, as claimed in (3.7.2). This finishes the proof of Theorem 3.7.

\section{REFERENCES}

[Alu06] Paolo Aluffi. Classes de Chern des variétés singulières, revisitées. C. R. Math. Acad. Sci. Paris, 342(6):405-410, 2006. $\uparrow 11$

[Amb05] Florin Ambro. The moduli b-divisor of an lc-trivial fibration. Compos. Math., 141(2):385-403, 2005. $\uparrow 6,24$

[BS94] Shigetoshi Bando and Yum-Tong Siu. Stable sheaves and Einstein-Hermitian metrics. In Geometry and analysis on complex manifolds, pages 39-50. World Sci. Publ., River Edge, NJ, 1994. $\uparrow 5$ 
[BS95] Mauro C. Beltrametti and Andrew J. Sommese. The adjunction theory of complex projective varieties, volume 16 of de Gruyter Expositions in Mathematics. Walter de Gruyter \& Co., Berlin, 1995. $\uparrow 15$

[Cat07] Fabrizio Catanese. Q.E.D. for algebraic varieties. J. Differential Geom., 77(1):43-75, 2007. With an appendix by Sönke Rollenske. $\uparrow 2$

[Del70] Pierre Deligne. Équations différentielles à points singuliers réguliers. Springer-Verlag, Berlin, 1970. Lecture Notes in Mathematics, Vol. 163. $\uparrow 4,21$

[DG94] Gerd Dethloff and Hans Grauert. Seminormal complex spaces. In Several Complex Variables VII, volume 74 of Encyclopaedia Math. Sci., pages 183-220. Springer, Berlin, 1994. $\uparrow 10,11$

[Dim92] Alexandru Dimca. Singularities and topology of hypersurfaces. Universitext. Springer-Verlag, New York, 1992. $\uparrow 17$

[Don85] Simon K. Donaldson. Anti self-dual Yang-Mills connections over complex algebraic surfaces and stable vector bundles. Proc. London Math. Soc. (3), 50(1):1-26, 1985. 24

[Dré04] Jean-Marc Drézet. Luna's slice theorem and applications. In Algebraic group actions and quotients, pages 39-89. Hindawi Publ. Corp., Cairo, 2004. $\uparrow 9$

[Dru14] Stéphane Druel. The Zariski-Lipman conjecture for log canonical spaces. Bull. London Math. Soc. 46(4):827-835, 2014. DOI:10.1112/blms /bdu040. $\uparrow 22$

[FL81] William Fulton and Robert Lazarsfeld. Connectivity and its applications in algebraic geometry. In Algebraic geometry (Chicago, Ill., 1980), volume 862 of Lecture Notes in Math., pages 26-92. Springer, Berlin, 1981. $\uparrow 18$

[Fle84] Hubert Flenner. Restrictions of semistable bundles on projective varieties. Comment. Math. Helv., 59(4):635-650, 1984. DOI: 10.1007/BF02566370. $\uparrow 23$

[Ful98] William Fulton. Intersection Theory, volume 2 of Ergebnisse der Mathematik und ihrer Grenzgebiete. 3. Folge. A Series of Modern Surveys in Mathematics [Results in Mathematics and Related Areas. 3rd Series. A Series of Modern Surveys in Mathematics]. Springer-Verlag, Berlin, second edition, 1998. $\uparrow 12$

[GKKP11] Daniel Greb, Stefan Kebekus, Sándor J. Kovács, and Thomas Peternell. Differential forms on log canonical spaces. Inst. Hautes études Sci. Publ. Math., 114(1):87-169, November 2011. DOI:10.1007/s10240-011-0036-0 An extended version with additional graphics is available as arXiv:1003.2913. $\uparrow$ 4, 22

[GKP11] Daniel Greb, Stefan Kebekus, and Thomas Peternell. Singular spaces with trivial canonical class. Preprint arXiv:1110.5250. To appear in Minimal models and extremal rays - Proceedings of the conference in honor of Shigefumi Mori's 60th birthday, Advanced Studies in Pure Mathematics, Kinokuniya Publishing House, Tokyo., October 2011. $\uparrow 21,24,25$

[GM88] Mark Goresky and Robert D. MacPherson. Stratified Morse theory, volume 14 of Ergebnisse der Mathematik und ihrer Grenzgebiete (3) [Results in Mathematics and Related Areas (3)]. SpringerVerlag, Berlin, 1988. ^9, 23

[GR58] Hans Grauert and Reinhold Remmert. Komplexe Räume. Math. Ann., 136:245-318, 1958. $\uparrow 11$

[GR84] Hans Grauert and Reinhold Remmert. Coherent analytic sheaves, volume 265 of Grundlehren der Mathematischen Wissenschaften [Fundamental Principles of Mathematical Sciences]. SpringerVerlag, Berlin, 1984. $\uparrow 8,17$

[Gra15] Patrick Graf. Bogomolov-Sommese vanishing on log canonical pairs. J. Reine Angew. Math., 702:109-142, 2015. DOI: 10.1515/crelle-2013-0031. $\uparrow 22$

[Gro66] Alexandre Grothendieck. Éléments de géométrie algébrique. IV. Étude locale des schémas et des morphismes de schémas. Troisiéme partie. Inst. Hautes Études Sci. Publ. Math., (28):255, 1966. Revised in collaboration with Jean Dieudonné. Freely available on the Numdam web site at http://www.numdam.org/numdam-bin/feuilleter?id=PMIHES_1966_ _28_. 9 9, 15, 30

[Gro68] Alexander Grothendieck. Cohomologie locale des faisceaux cohérents et théorèmes de Lefschetz locaux et globaux (SGA 2). North-Holland Publishing Co., Amsterdam, 1968. Augmenté d'un exposé par Michèle Raynaud, Séminaire de Géométrie Algébrique du Bois-Marie, 1962, Advanced Studies in Pure Mathematics, Vol. 2. Also available as arXiv:math/0511279. $\uparrow 15$

[Gro70] Alexander Grothendieck. Représentations linéaires et compactification profinie des groupes discrets. Manuscripta Math., 2:375-396, 1970. $\uparrow 21$

[Gro71] Alexandre Grothendieck. Revêtements étales et groupe fondamental (SGA 1). Springer-Verlag, Berlin, 1971. Séminaire de Géométrie Algébrique du Bois Marie 1960-1961, Dirigé par Alexandre Grothendieck. Augmenté de deux exposés de Michèle Raynaud, Lecture Notes in Mathematics, Vol. 224. Also available as arXiv:math/0206203. $\uparrow 17,31$

[GZ94] Rajendra V. Gurjar and De-Qi Zhang. $\pi_{1}$ of smooth points of a log del Pezzo surface is finite. I. J. Math. Sci. Univ. Tokyo, 1(1):137-180, 1994. $\uparrow 27$

[Har77] Robin Hartshorne. Algebraic geometry. Springer-Verlag, New York, 1977. Graduate Texts in Mathematics, No. 52. $\uparrow 8,16,30,31$ 
[Har80] Robin Hartshorne. Stable reflexive sheaves. Math. Ann., 254(2):121-176, 1980. DOI: 10.1007/BF01467074. $\uparrow 15,16$

[Hat02] Allen Hatcher. Algebraic topology. Cambridge University Press, Cambridge, 2002. Available from the author's home page at http://www . math. cornell. edu/ hatcher. $\uparrow 8,10$

[HL10] Daniel Huybrechts and Manfred Lehn. The geometry of moduli spaces of sheaves. Cambridge Mathematical Library. Cambridge University Press, Cambridge, second edition, 2010. $\uparrow 23$

[HMX14] Christopher D. Hacon, James McKernan, and Chenyang Xu. ACC for log canonical thresholds. Ann. of Math. (2), 180(2):523-571, 2014. $\uparrow 4$

[KK10] Stefan Kebekus and Sándor J. Kovács. The structure of surfaces and threefolds mapping to the moduli stack of canonically polarized varieties. Duke Math. J., 155(1):1-33, 2010. $\uparrow 2$

[KM98] János Kollár and Shigefumi Mori. Birational geometry of algebraic varieties, volume 134 of Cambridge Tracts in Mathematics. Cambridge University Press, Cambridge, 1998. $\uparrow$ 5, 8, 21, $22,23,24$

[Kob87] Shoshichi Kobayashi. Differential geometry of complex vector bundles, volume 15 of Publications of the Mathematical Society of Japan. Iwanami Shoten and Princeton University Press, Princeton, NJ, 1987. Kanô Memorial Lectures, 5. $\uparrow$ 5, 22

[Ko195] János Kollár. Shafarevich maps and automorphic forms. M. B. Porter Lectures. Princeton University Press, Princeton, NJ, 1995. $\uparrow 18,22,28$

[LB70] Alain Lascoux and Marcel Berger. Variétés Kähleriennes compactes. Lecture Notes in Mathematics, Vol. 154. Springer-Verlag, Berlin, 1970. $\uparrow 5$

[Mac74] Robert D. MacPherson. Chern classes for singular algebraic varieties. Ann. of Math. (2), 100:423-432, 1974. $\uparrow 11$

[Mil80] James S. Milne. Étale cohomology, volume 33 of Princeton Mathematical Series. Princeton University Press, Princeton, N.J., 1980. $\uparrow 3,8,18,31$

[Miy87] Yoichi Miyaoka. The Chern classes and Kodaira dimension of a minimal variety. In Algebraic geometry, Sendai, 1985, volume 10 of Adv. Stud. Pure Math., pages 449-476. North-Holland, Amsterdam, 1987. $\uparrow 14$

[Mum08] David Mumford. Abelian varieties, volume 5 of Tata Institute of Fundamental Research Studies in Mathematics. Published for the Tata Institute of Fundamental Research, Bombay, 2008. With appendices by C. P. Ramanujam and Yuri Manin, Corrected reprint of the second (1974) edition. $\uparrow 31$

[NZ10] Noboru Nakayama and De-Qi Zhang. Polarized endomorphisms of complex normal varieties. Math. Ann., 346(4):991-1018, 2010. $\uparrow 6,25,26$

[Ros68] Hugo Rossi. Picard variety of an isolated singular point. Rice Univ. Studies, 54(4):63-73, 1968. $\uparrow 11$

[RZ10] Luis Ribes and Pavel Zalesskii. Profinite groups, volume 40 of Ergebnisse der Mathematik und ihrer Grenzgebiete. 3. Folge. A Series of Modern Surveys in Mathematics [Results in Mathematics and Related Areas. 3rd Series. A Series of Modern Surveys in Mathematics]. Springer-Verlag, Berlin, second edition, 2010. $\uparrow 18,21,22$

[SBW94] Nicholas I. Shepherd-Barron and Pelham M.H. Wilson. Singular threefolds with numerically trivial first and second Chern classes. J. Algebraic Geom., 3(2):265-281, 1994. $\uparrow$ 5, 24

[Sim92] Carlos T. Simpson. Higgs bundles and local systems. Inst. Hautes Études Sci. Publ. Math., 75:5-95, 1992. $\uparrow 5,23,24$

[Sim94] Carlos T. Simpson. Moduli of representations of the fundamental group of a smooth projective variety. II. Inst. Hautes Études Sci. Publ. Math., 80:5-79 (1995), 1994. ^ 6, 23

[Ste56] Karl Stein. Analytische Zerlegungen komplexer Räume. Math. Ann., 132:63-93, $1956 . \uparrow 11$

[Tak00] Shigeharu Takayama. Simple connectedness of weak Fano varieties. J. Algebraic Geom., 9(2):403-407, 2000. $\uparrow 21$

[UY86] Karen Uhlenbeck and Shing-Tung Yau. On the existence of Hermitian-Yang-Mills connections in stable vector bundles. Comm. Pure Appl. Math., 39(S, suppl.):S257-S293, 1986. Frontiers of the mathematical sciences: 1985 (New York, 1985). $\uparrow 5$

[Weh73] Bertram A. F. Wehrfritz. Infinite linear groups. An account of the group-theoretic properties of infinite groups of matrices. Springer-Verlag, New York, 1973. Ergebnisse der Matematik und ihrer Grenzgebiete, Band 76. $\uparrow 22$

[Wei94] Charles A. Weibel. An introduction to homological algebra, volume 38 of Cambridge Studies in Advanced Mathematics. Cambridge University Press, Cambridge, 1994. $\uparrow 16$

[Xu14] Chenyang Xu. Finiteness of algebraic fundamental groups. Compositio Math., 150(3):409414, 2014. $\uparrow 4,17$

[Yau78] Shing Tung Yau. On the Ricci curvature of a compact Kähler manifold and the complex Monge-Ampère equation. I. Comm. Pure Appl. Math., 31(3):339-411, 1978. 5

[Zha06] Qi Zhang. Rational connectedness of log Q-Fano varieties. J. Reine Angew. Math., 590:131142, 2006. $\uparrow 21$ 
Daniel Greb, Essener Seminar für Algebraische GeOMetrie und ARITHMetik, FaKUltät FÜR MATHEMATIK, UNIVERSITÄt DUISBURG-ESSEN, 45117 ESSEN, GERMANY

E-mail address: daniel.greb@uni-due.de

URL: http://www.esaga.uni-due.de/daniel.greb

Stefan Kebekus, Mathematisches institut, Albert-Ludwigs-Universität Freiburg, ECKERSTRASSE 1, 79104 FREIBURG IM BREISGAU, GERMANY AND UNIVERSITY OF STRASBOURG INSTITUTE FOR ADVANCED STUdy (USIAS), STRASBOURG, FRANCE

E-mail address: stef an.kebekus@math.uni-freiburg.de

URL: http://home.mathematik.uni-freiburg.de/kebekus

Thomas Peternell, Mathematisches Institut, Universität Bayreuth, 95440 Bayreuth, GERMANY

E-mail address: thomas.peternell@uni-bayreuth.de

URL: http://www.staff .uni-bayreuth.de/ btm109/peternell/pet.html 